ESAIM: M2AN 50 (2016) 1763-1787

DOI: $10.1051 / \mathrm{m} 2 \mathrm{an} / 2016006$
ESAIM: Mathematical Modelling and Numerical Analysis

www.esaim-m2an.org

\title{
MAXWELL'S EQUATIONS FOR CONDUCTORS WITH IMPEDANCE BOUNDARY CONDITIONS: DISCONTINUOUS GALERKIN AND REDUCED BASIS METHODS *
}

\author{
Kristin Kirchner ${ }^{1}$, Karsten Urban ${ }^{2}$ and Oliver Zeeb ${ }^{2}$
}

\begin{abstract}
We consider Maxwell's equations with impedance boundary conditions on a conductive polyhedron with polyhedral holes. Well-posedness of the variational formulation is proven, a hpdiscontinuous Galerkin (hp-dG) approximation as well as a priori error estimates are introduced. Next, we use the frequency $\omega$ as a parameter in a multi-query context. For this purpose, we derive a Reduced Basis Method (RBM) based upon the dG formulation as well as the corresponding a posteriori error bound. Numerical results indicate the efficiency and the robustness of the scheme.
\end{abstract}

Mathematics Subject Classification. 35Q61, 65N30, 65N15.

Received July 2, 2015. Revised November 30, 2015. Accepted January 22, 2016.

\section{INTRODUCTION}

This paper is concerned with the analysis and the efficient numerical solution of the time-harmonic Maxwell's equations on a simply-connected conductive polyhedron which may have polyhedral holes. These holes can be seen as rigid bodies of perfectly conducting material, whereas on the exterior we impose an impedance boundary condition. In particular, we are interested in solving such a problem rapidly and certified by an a posteriori error control for several different values of the frequency $\omega$.

We start by proving well-posedness of the variational formulation in Section 2. Due to our assumption of positive electric conductivity $\sigma \geq \sigma_{-}>0$, this is a more or less standard application of the Lax-Milgram theorem. However, verifying coercivity and boundedness is not trivial under our (mild) assumptions and we were not able to find a corresponding proof in the literature. For this reason and in order to derive explicit bounds for the constants in terms of the parameter $\omega$, we detail all arguments in Appendix A.

Next, in Section 3, we construct a hp-discontinuous Galerkin (hp-dG) numerical method to obtain a discretization of the electric field density $\boldsymbol{E}$. We detail well-posedness, which is again not trivially seen, and the a priori convergence analysis. These results are a generalization of [22]. Specifically, in Section 3.1 we use an

Keywords and phrases. Maxwell's equations, impedance, conductor, discontinuous Galerkin, reduced Basis Method.

* K.U. and O.Z. gratefully acknowledge support by Deutsche Forschungsgemeinschaft GrK1100.

1 Department of Mathematical Sciences, Chalmers University of Technology and University of Gothenburg, SE-412 96 Gothenburg, Sweden. kristin.kirchner@chalmers.se

2 University of Ulm, Institute for Numerical Mathematics, Helmholtzstrasse 18, 89069 Ulm, Germany.

karsten.urban@uni-ulm.de; oliver.zeeb@uni-ulm.de 
interior penalty $\mathrm{dG}$ flux and derive a consistent discrete variational formulation in the nonconforming space of piecewise polynomial functions. The corresponding sesquilinear form is shown to be continuous and coercive w.r.t. an appropriate energy norm in Section 3.2. This provides us with the foundation for our error analysis in Section 3.3, where we show convergence at an optimal rate w.r.t. the energy norm.

As already pointed out, our main objective is to solve the time-harmonic Maxwell problem for several different values of the frequency $\omega$. Hence, we obtain parameterized Maxwell's equations, where we seek a numerical approximation for many values of the frequency. For this kind of multi-query problems, i.e., solving the same problem for many different values of the parameter, the Reduced Basis Method (RBM) has become a well-accepted efficient numerical scheme, in particular for parameterized partial differential equations (pde's). Roughly speaking, the RBM is based upon a separation into offline and online computations and on the availability of a detailed,but possibly costly numerical model, e.g., with a fine mesh size $h$ and a huge number $\mathcal{N}=\mathcal{N}_{h}$ of unknowns. Using this detailed model and an efficiently computable error bound allows one to determine parameter values, say $\omega_{1}, \ldots, \omega_{N}, N \ll \mathcal{N}$, in the offline phase by maximizing the error estimator w.r.t. the parameter $\omega^{3}$. For these $\omega_{i}$, the detailed solution $\boldsymbol{\xi}_{i}:=\boldsymbol{E}_{h}\left(\omega_{i}\right)$ is computed in the offline phase and stored. Then, the set $\left\{\boldsymbol{\xi}_{1}, \ldots, \boldsymbol{\xi}_{N}\right\}$ is called reduced basis, which is used in the online phase to compute an approximation $\boldsymbol{E}_{N}(\omega)$ for a new parameter value $\omega \neq \omega_{i}$. The already mentioned a posteriori error bound gives rise to a certified reduced numerical approximation.

There are several articles dealing with RBMs for different versions of Maxwell's equations, see $[10,11,15,16$, $19,20,29]$, just to mention a few. However, to the best of our knowledge, the case treated in this paper has not been considered so far. For the following reasons we think that the presented framework is particularly interesting:

- The domain $\Omega$, on which we consider the pde, is non-convex and we allow for coefficients with low regularity $\left(\varepsilon, \sigma \in L^{\infty}(\Omega ; \mathbb{R}), \mu^{-1} \in W^{1, \infty}(\Omega ; \mathbb{R})\right)$, so that the solution cannot be expected to have maximal regularity and hence $H^{1}$-conforming finite elements may not be appropriate, whereas a dG approach seems adequate.

- Changing the frequency $\omega$, i.e., interpreting it as a parameter may also change the mathematical properties of the pde. If "critical" parameter values are not known a priori, RBM variants such as local RBM [24] or hp-RBM $[13,14]$ are at least not straightforward to apply. Such a strong parameter dependence as considered here is a crucial issue for a RBM.

- In the literature, usually perfectly conducting material and corresponding boundary conditions on all of $\partial \Omega$ have been considered. Instead, we use impedance boundary conditions on the outward part $\Sigma$ of $\partial \Omega$, see below.

Section 4 contains construction and analysis of a RBM for the above Maxwell setting. Finally, Section 5 is devoted to our numerical results that show efficiency and robustness of our approach. We collect details of certain proofs in Appendix A and the data for reproducing our numerical experiments in Appendix B.

\section{MODEL PROBLEM}

We consider an electromagnetic cavity problem on a bounded, simply-connected Lipschitz polyhedron $\Omega \subset$ $\mathbb{R}^{3}$ with $M$ disjoint connected boundary parts $\Gamma_{1}, \ldots, \Gamma_{M-1}, \Sigma$. Note that $\Sigma$ is the boundary of the only unbounded connected component of the complement $\mathbb{R}^{3} \backslash \bar{\Omega}$ - the "interface" to the exterior. At the interior boundaries $\Gamma_{1}, \ldots, \Gamma_{M-1}$ the domain $\Omega$ is assumed to be surrounded by perfectly conducting material. At the exterior boundary $\Sigma$ the electromagnetic field satisfies an impedance boundary condition. Following the approach presented, e.g., in [26] we obtain the following boundary value problem for the case of a time-harmonic

\footnotetext{
${ }^{3}$ In the RBM literature, usually the letter $\mu$ is used for denoting the parameter. Since we consider the frequency $\omega$ as the relevant parameter and here $\mu$ denotes the magnetic permeability, we use $\omega$ to denote the parameter.
} 


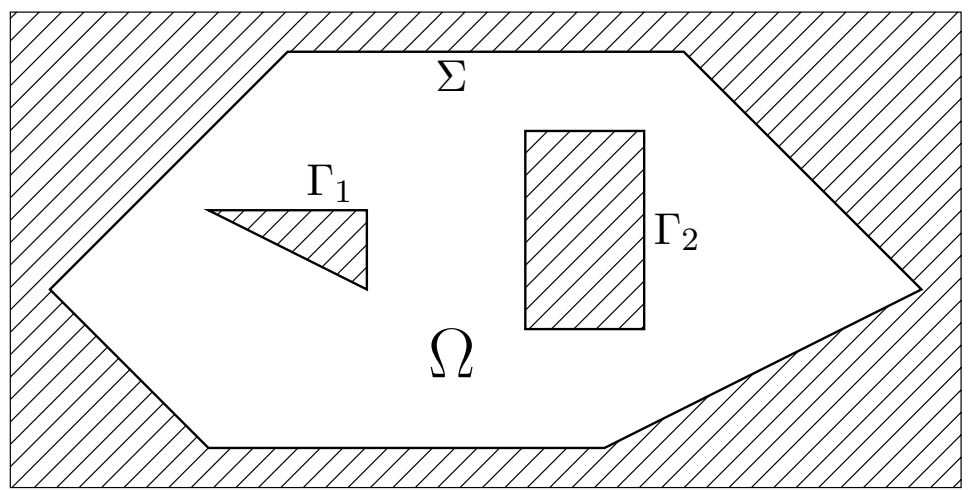

Figure 1. Cross section of a possible 3d-domain $\Omega$.

electromagnetic wave propagation ( $\boldsymbol{n}$ being the outward normal)

$$
\begin{aligned}
\nabla \times\left(\mu^{-1} \nabla \times \boldsymbol{E}\right)-\left(\omega^{2} \varepsilon+i \omega \sigma\right) \boldsymbol{E} & =i \omega \sqrt{\varepsilon_{0}} \boldsymbol{J}_{\boldsymbol{a}} & & \text { in } \Omega, \\
\boldsymbol{n} \times \boldsymbol{E} & =0 & & \text { on } \Gamma_{1}, \ldots, \Gamma_{M-1}, \\
\left(\mu^{-1} \nabla \times \boldsymbol{E}\right) \times \boldsymbol{n}-i \omega \lambda\left(\varepsilon_{0} \mu_{0}^{-1}\right)^{1 / 2} \boldsymbol{E}_{T} & =\mu_{0}^{-1} \boldsymbol{g} & & \text { on } \Sigma .
\end{aligned}
$$

As usual, $\mu_{0}=4 \pi \times 10^{-7} \mathrm{H} \mathrm{m}^{-1}$ and $\varepsilon_{0} \approx 8.854 \times 10^{-12} \mathrm{~F} \mathrm{~m}^{-1}$ denote the magnetic permeability and electric permittivity in vacuum, respectively. For $\boldsymbol{u} \in H(\operatorname{curl}, \Omega):=\left\{\boldsymbol{v} \in L^{2}\left(\Omega ; \mathbb{C}^{3}\right) \mid \nabla \times \boldsymbol{v} \in L^{2}\left(\Omega ; \mathbb{C}^{3}\right)\right\}$ the "tangential components trace" $\boldsymbol{u}_{T}$ on $\partial \Omega$ is defined as $\boldsymbol{u}_{T}:=\left(\boldsymbol{n} \times\left.\boldsymbol{u}\right|_{\partial \Omega}\right) \times \boldsymbol{n},(c f$. [8], Thm. 3.31 of [26]). Finally, $\lambda>0$ is a constant parameter depending on the intensity of the impedance. In addition, we make the following assumptions on the model.

\section{Assumption 2.1.}

(a) The magnetic permeability $\mu$ satisfies $\mu^{-1} \in W^{1, \infty}(\Omega ; \mathbb{R})$ and there exist constants $\mu_{-}, \mu_{+}>0$ such that

$$
0<\mu_{-} \leq \operatorname{ess} \inf _{\boldsymbol{x} \in \Omega} \mu(\boldsymbol{x}) \leq \operatorname{ess} \sup _{\boldsymbol{x} \in \Omega} \mu(\boldsymbol{x}) \leq \mu_{+}<+\infty .
$$

(b) We assume $\varepsilon, \sigma \in L^{\infty}(\Omega ; \mathbb{R})$ and that $\Omega$ is a conductor, i.e, there exist constants $\varepsilon_{-}, \varepsilon_{+}, \sigma_{-}, \sigma_{+}>0$ with

$$
\begin{aligned}
& 0<\varepsilon_{-} \leq \operatorname{ess} \inf _{\boldsymbol{x} \in \Omega} \varepsilon(\boldsymbol{x}) \leq \operatorname{ess} \sup _{\boldsymbol{x} \in \Omega} \varepsilon(\boldsymbol{x}) \leq \varepsilon_{+}<+\infty, \\
& 0<\sigma_{-} \leq \operatorname{ess} \inf _{\boldsymbol{x} \in \Omega} \sigma(\boldsymbol{x}) \leq \operatorname{ess} \sup _{\boldsymbol{x} \in \Omega} \sigma(\boldsymbol{x}) \leq \sigma_{+}<+\infty .
\end{aligned}
$$

(c) $\boldsymbol{J}_{\boldsymbol{a}} \in H(\operatorname{div}, \Omega):=\left\{\boldsymbol{u} \in L^{2}\left(\Omega ; \mathbb{C}^{3}\right) \mid \nabla \cdot \boldsymbol{u} \in L^{2}(\Omega ; \mathbb{C})\right\}$.

(d) $\boldsymbol{g} \in L_{t}^{2}\left(\Sigma ; \mathbb{C}^{3}\right):=\left\{\boldsymbol{v} \in L^{2}\left(\Sigma ; \mathbb{C}^{3}\right) \mid \boldsymbol{n} \cdot \boldsymbol{v}=0\right.$ a.e. on $\left.\Sigma\right\}$.

Remark 2.2. We point out assumption (2.4), which states that the electric conductivity is assumed to be positive and bounded away from zero. This is indeed crucial for the subsequent analysis as it ensures coercivity.

Proceeding as in [26], Chapter 4, a variational formulation of (2.1a)-(2.1c) reads: given $\omega>0$, find $\boldsymbol{E} \in X$ such that

$$
a_{e}(\boldsymbol{E}, \boldsymbol{v} ; \omega)=f(\boldsymbol{v} ; \omega) \quad \forall \boldsymbol{v} \in X
$$


with the trial and test space defined as

$$
X:=\left\{\boldsymbol{u} \in H(\operatorname{curl}, \Omega) \mid \boldsymbol{n} \times \boldsymbol{u}=0 \text { on } \Gamma_{1}, \ldots, \Gamma_{M-1} ; \boldsymbol{u}_{T} \in L^{2}\left(\Sigma ; \mathbb{C}^{3}\right) \text { on } \Sigma\right\},
$$

the sesquilinear form

$$
\begin{aligned}
a_{e}(\boldsymbol{E}, \boldsymbol{v} ; \omega):= & \left(\mu^{-1} \nabla \times \boldsymbol{E}, \nabla \times \boldsymbol{v}\right)_{\Omega}-\omega^{2}(\varepsilon \boldsymbol{E}, \boldsymbol{v})_{\Omega}-i \omega(\sigma \boldsymbol{E}, \boldsymbol{v})_{\Omega} \\
& -i \omega \lambda\left(\varepsilon_{0} \mu_{0}^{-1}\right)^{1 / 2}\left\langle\boldsymbol{E}_{T}, \boldsymbol{v}_{T}\right\rangle_{\Sigma}
\end{aligned}
$$

and the right-hand side

$$
f(\boldsymbol{v} ; \omega):=i \omega \sqrt{\varepsilon_{0}}\left(\boldsymbol{J}_{\boldsymbol{a}}, \boldsymbol{v}\right)_{\Omega}+\mu_{0}^{-1}\left\langle\boldsymbol{g}, \boldsymbol{v}_{T}\right\rangle_{\Sigma},
$$

where $(\cdot, \cdot)_{\Omega}$ denotes the inner product on $L^{2}\left(\Omega ; \mathbb{C}^{3}\right)$ and $\left\langle\cdot, \cdot_{T}\right\rangle_{\Sigma}$ is the dual pairing w.r.t. the pivot space $L^{2}\left(\Sigma, \mathbb{C}^{3}\right)$, i.e., $\left\langle\boldsymbol{g}, \boldsymbol{v}_{T}\right\rangle_{\Sigma}:=\int_{\Sigma} \boldsymbol{g} \cdot \overline{\boldsymbol{v}}_{T} \mathrm{~d} S$ for $\boldsymbol{g} \in L_{t}^{2}\left(\Sigma ; \mathbb{C}^{3}\right), \boldsymbol{v} \in H(\operatorname{curl}, \Omega)$. As shown in ([26], Thm. 4.1), $X$ is a Hilbert space with inner product $(\boldsymbol{u}, \boldsymbol{v})_{X}:=(\nabla \times \boldsymbol{u}, \nabla \times \boldsymbol{v})_{\Omega}+(\boldsymbol{u}, \boldsymbol{v})_{\Omega}+\left\langle\boldsymbol{u}_{T}, \boldsymbol{v}_{T}\right\rangle_{\Sigma}, \boldsymbol{u}, \boldsymbol{v} \in X$, and induced norm $\|\boldsymbol{u}\|_{X}^{2}:=(\boldsymbol{u}, \boldsymbol{u})_{X}$. It seems natural that (2.5) is well-posed in conductive mediums, i.e., if $\sigma \geq \sigma_{-}>0$. However, to the best of our knowledge there is no proof in the literature dealing with regularity assumptions on the coefficients $\mu, \varepsilon$ and $\sigma$ which are as low as in our case. Therefore, we include it in Appendix A.

Since we investigate problem (2.5) in a context with $\omega$ as a parameter, we state the dependency of all constants in terms of $\omega$.

Theorem 2.3 (Existence and uniqueness of $\boldsymbol{E}$ ). Suppose Assumption 2.1 is satisfied. Then, there exists a unique solution $\boldsymbol{E}(\omega) \in X$ to (2.5). In particular, the bilinear form $a_{e}(\cdot, \cdot)$ in $(2.7)$ is continuous and coercive with constants

$$
\begin{aligned}
\gamma(\omega) & :=\max \left\{\mu_{-}^{-1}, \omega^{2} \varepsilon_{+}+\omega \sigma_{+}, \omega \lambda\left(\varepsilon_{0} \mu_{0}^{-1}\right)^{1 / 2}\right\}, \\
\alpha(\omega) & :=\min \left\{\frac{\sigma_{-}}{2^{3 / 2} \mu_{+}\left(\omega^{2} \varepsilon_{+}^{2}+\sigma_{-}^{2}\right)^{1 / 2}}, \frac{\omega}{2}\left(\frac{\omega^{2} \varepsilon_{+}^{2} \sigma_{-}^{2}+\sigma_{-}^{4}}{2 \omega^{2} \varepsilon_{+}^{2}+\sigma_{-}^{2}}\right)^{\frac{1}{2}}, \frac{\omega \lambda \sqrt{\varepsilon_{0}}}{\sqrt{2 \mu_{0}}}\right\} .
\end{aligned}
$$

The right-hand side (2.8) is bounded by $\|f(\cdot ; \omega)\|_{X^{\prime}} \leq C_{f}(\omega):=\omega \sqrt{\varepsilon_{0}}\left\|\boldsymbol{J}_{\boldsymbol{a}}\right\|_{L^{2}(\Omega)^{3}}+\mu_{0}^{-1}\|\boldsymbol{g}\|_{L^{2}(\Sigma)^{3}}$. In particular, the stability estimate $\|\boldsymbol{E}(\omega)\|_{X} \leq \frac{C_{f}(\omega)}{\alpha(\omega)}$ holds.

Proof. The claim follows from a complex-valued version of the Lax-Milgram lemma, (e.g. [26], Lem. 2.21), since $a_{e}: X \times X \rightarrow \mathbb{C}$ in (2.7) is continuous and coercive and $f: X \rightarrow \mathbb{C}$ in (2.8) is bounded, which is shown in detail in Appendix A.1.

\section{Discontinuous Galerkin approximation}

In this section we introduce a hp-discontinuous Galerkin (hp-dG) formulation of (2.1a)-(2.1c) resp. (2.5). For this purpose, we adapt an interior penalty numerical flux in [22] (there for the special case of a perfectly conducting boundary, $\boldsymbol{n} \times \boldsymbol{E}=0$ on all of $\partial \Omega$, and constant material parameters $\mu \equiv \mu_{0}, \varepsilon \equiv \varepsilon_{0}$ and $\sigma \equiv 0$ ).

\subsection{Interior penalty $\mathrm{dG}$ formulation}

The derivation of the $\mathrm{dG}$ formulation follows the ideas of [5], where a general $\mathrm{dG}$ approach for elliptic problems using different numerical fluxes is described. Instead of the Laplace operator we have to investigate the curl-curl operator. 
First, we introduce the auxiliary function $\boldsymbol{q} \in L^{2}\left(\Omega ; \mathbb{C}^{3}\right)$ satisfying $\mu \boldsymbol{q}=\nabla \times \boldsymbol{E}$ a.e. in $\Omega$, so that instead of (2.1a) $-(2.1 \mathrm{c})$ we can consider the first-order system

$$
\begin{aligned}
\nabla \times \boldsymbol{q}-\left(\omega^{2} \varepsilon+i \omega \sigma\right) \boldsymbol{E} & =i \omega \sqrt{\varepsilon_{0}} \boldsymbol{J}_{\boldsymbol{a}} & & \text { in } \Omega, \\
\mu \boldsymbol{q} & =\nabla \times \boldsymbol{E} & & \text { in } \Omega, \\
\boldsymbol{n} \times \boldsymbol{E} & =0 & & \text { on } \Gamma_{1}, \ldots, \Gamma_{M-1}, \\
\boldsymbol{q} \times \boldsymbol{n}-i \omega \lambda\left(\varepsilon_{0} \mu_{0}^{-1}\right)^{1 / 2} \boldsymbol{E}_{T} & =\mu_{0}^{-1} \boldsymbol{g} & & \text { on } \Sigma .
\end{aligned}
$$

We follow the standard discontinuous Galerkin approach, namely: (1) partition of the polyhedron $\Omega$ into a finite set of elements; (2) multiply (3.1a) and (3.1b) with test functions, integrate over $\Omega$ and use integration by parts on each element; (3) in the integrals over the elemental boundaries replace $\boldsymbol{q}$ and $\boldsymbol{E}$ by their numerical fluxes $\boldsymbol{q}_{h}^{*}$ and $\boldsymbol{E}_{h}^{*} ;(4)$ again, integrate $(3.1 \mathrm{~b})$ by parts on each element.

Although this dG construction is standard, to the best of our knowledge it has not been applied to the Maxwell problem with impedance boundary conditions before. In addition, the regularity assumptions that we impose on the coefficients $\mu, \varepsilon$ and $\sigma$ as well as on the exact solution $\boldsymbol{E}$ are quite low so that a careful analysis is needed not only in order to prove convergence of the $\mathrm{dG}$ scheme, but also as a preliminary for the subsequent RBM.

As for (1), let $\mathcal{T}_{h}$ be a shape-regular mesh of tetrahedra covering the polyhedral domain $\Omega$. For each element $T \in \mathcal{T}_{h}$ we define $h_{T}$ as the diameter of the smallest sphere containing $T$, and for $\mathcal{T}_{h}$ we define the mesh size as $h:=\max _{T \in \mathcal{T}_{h}} h_{T}$. Furthermore, let $\mathcal{F}_{h}$ denote the set of all faces in $\mathcal{T}_{h}, \mathcal{F}_{h}^{I}:=\left\{F \in \mathcal{F}_{h}: F \subset \Omega\right\}$ and $\mathcal{F}_{h}^{B}:=$ $\left\{F \in \mathcal{F}_{h}: F \subset \partial \Omega\right\}$ the set of all interior and boundary faces, respectively. We partition $\mathcal{F}_{h}^{B}$ in accordance to the two different boundary conditions (3.1c) and (3.1d), i.e., $\mathcal{F}_{h}^{\Gamma}:=\left\{F \in \mathcal{F}_{h}: F \subset\left(\Gamma_{1} \cup \ldots \cup \Gamma_{M-1}\right)\right\}$, $\mathcal{F}_{h}^{\Sigma}:=\left\{F \in \mathcal{F}_{h}: F \subset \Sigma\right\}$. The size of each face $F \in \mathcal{F}_{h}$ is measured by the diameter $h_{F}$ of the smallest circle containing $F$. In this context, we additionally define the function $\mathrm{h}$ by

$$
\mathrm{h}: \bigcup_{F \in \mathcal{F}_{h}} F \rightarrow \mathbb{R}_{>0}, \quad \mathrm{~h}(\boldsymbol{x}):=\sum_{F \in \mathcal{F}_{h}} h_{F} \chi_{F}(\boldsymbol{x}) .
$$

For the dG formulation we need the following definitions of the tangential jump and average across an interface $F \in \mathcal{F}_{h}$ between two tetrahedra $T^{L} \neq T^{R}$, which are well-defined for all functions $\boldsymbol{u}$ satisfying $\boldsymbol{u} \in\left\{\boldsymbol{f} \in L^{2}\left(\Omega ; \mathbb{C}^{3}\right)|\boldsymbol{f}|_{T} \in C^{0}\left(T ; \mathbb{C}^{3}\right) \forall T \in \mathcal{T}_{h}\right\}$,

$$
\begin{aligned}
\llbracket \boldsymbol{u} \rrbracket: & = \begin{cases}\boldsymbol{n}_{T^{L}} \times\left.\boldsymbol{u}\right|_{T^{L}}+\boldsymbol{n}_{T^{R}} \times\left.\boldsymbol{u}\right|_{T^{R}} & \text { on } F \in \mathcal{F}_{h}^{I}, F \subset \partial T^{L} \cap \partial T^{R}, \\
\boldsymbol{n} \times \boldsymbol{u} & \text { on } F \in \mathcal{F}_{h}^{B},\end{cases} \\
\{\{\boldsymbol{u}\}] & := \begin{cases}\frac{1}{2}\left(\left.\boldsymbol{u}\right|_{T^{L}}+\left.\boldsymbol{u}\right|_{T^{R}}\right) & \text { on } F \in \mathcal{F}_{h}^{I}, F \subset \partial T^{L} \cap \partial T^{R}, \\
\boldsymbol{u} & \text { on } F \in \mathcal{F}_{h}^{B} .\end{cases}
\end{aligned}
$$

Here, $\boldsymbol{n}_{T^{L}}$ denotes the outward normal of $T^{L}, \boldsymbol{n}_{T^{R}}$ the one of $T^{R}$. In this context, we recall the so-called "dG magic formula", (cf. [21], Eq. (3.1)),

$$
\sum_{T \in \mathcal{T}_{h}}\left\langle\boldsymbol{n}_{T} \times \boldsymbol{u}, \boldsymbol{v}\right\rangle_{\partial T}=\sum_{F \in \mathcal{F}_{h}^{I}}\langle\llbracket \boldsymbol{u} \rrbracket,\{\{\boldsymbol{v}\}\}\rangle_{F}-\sum_{F \in \mathcal{F}_{h}^{I}}\langle\{\{\boldsymbol{u}\}\}, \llbracket \boldsymbol{v} \rrbracket\rangle_{F}+\sum_{F \in \mathcal{F}_{h}^{B}}\langle\boldsymbol{n} \times \boldsymbol{u}, \boldsymbol{v}\rangle_{F}
$$

for $\boldsymbol{u}, \boldsymbol{v} \in\left\{\boldsymbol{f} \in L^{2}\left(\Omega ; \mathbb{C}^{3}\right)|\boldsymbol{f}|_{T} \in C^{0}\left(T, \mathbb{C}^{3}\right) \forall T \in \mathcal{T}_{h}\right\}$, where $\langle\cdot, \cdot\rangle_{\partial T}$ and $\langle\cdot, \cdot\rangle_{F}$ are the dual pairings on element boundaries and interfaces induced by $L^{2}\left(\partial T ; \mathbb{C}^{3}\right)$ and $L^{2}\left(F ; \mathbb{C}^{3}\right)$, respectively. In order to shorten notation, we abbreviate:

$$
\begin{aligned}
\langle\cdot, \cdot\rangle_{\mathcal{F}_{h}}:=\sum_{F \in \mathcal{F}_{h}}\langle\cdot, \cdot\rangle_{F}, & \langle\cdot, \cdot\rangle_{\mathcal{F}_{h}^{B}}:=\sum_{F \in \mathcal{F}_{h}^{B}}\langle\cdot, \cdot\rangle_{F}, & \langle\cdot, \cdot\rangle_{\mathcal{F}_{h}^{I}}:=\sum_{F \in \mathcal{F}_{h}^{I}}\langle\cdot, \cdot\rangle_{F}, \\
\langle\cdot, \cdot\rangle_{\mathcal{F}_{h}}:=\sum_{F \in \mathcal{F}_{h}^{D}}\langle\cdot, \cdot\rangle_{F}, & \langle\cdot, \cdot\rangle_{\mathcal{F}_{h}^{I}}:=\sum_{F \in \mathcal{F}_{h}^{I}}\langle\cdot, \cdot\rangle_{F}, & \langle\cdot, \cdot\rangle_{\mathcal{F}_{h}^{I \cup I}}:=\sum_{F \in \mathcal{F}_{h}^{I} \cup \mathcal{F}_{h}^{I}}\langle\cdot, \cdot\rangle_{F} .
\end{aligned}
$$


Next, for (2) above, we multiply (3.1a) with the complex conjugate of

$$
\boldsymbol{v}_{h} \in V_{h, p}:=\left\{\boldsymbol{\psi} \in L^{2}\left(\Omega ; \mathbb{C}^{3}\right)|\boldsymbol{\psi}|_{T} \in \mathcal{P}^{p}(T ; \mathbb{C})^{3} \forall T \in \mathcal{T}_{h}\right\}
$$

where $\mathcal{P}^{p}(T ; \mathbb{C})$ denotes the space of complex-valued polynomials of degree at most $p \in \mathbb{N}$ on $T$. Replacing $\boldsymbol{q}$ and $\boldsymbol{E}$ with approximations $\boldsymbol{q}_{h}, \boldsymbol{E}_{h} \in V_{h, p}$ and integrating over $\Omega$ yields

$$
\left(\nabla_{h} \times \boldsymbol{q}_{h}, \boldsymbol{v}_{h}\right)_{\Omega}-\omega^{2}\left(\varepsilon \boldsymbol{E}_{h}, \boldsymbol{v}_{h}\right)_{\Omega}-i \omega\left(\sigma \boldsymbol{E}_{h}, \boldsymbol{v}_{h}\right)_{\Omega}=i \omega \sqrt{\varepsilon_{0}}\left(\boldsymbol{J}_{\boldsymbol{a}}, \boldsymbol{v}_{h}\right)_{\Omega}
$$

Here, $\nabla_{h} \times$ denotes the elementwise curl operator for functions in $V_{h, p}$, i.e., $\left(\nabla_{h} \times \boldsymbol{q}_{h}, \boldsymbol{v}_{h}\right)_{\Omega}:=\sum_{T \in \mathcal{T}_{h}}(\nabla \times$ $\left.\boldsymbol{q}_{h}, \boldsymbol{v}_{h}\right)_{T}$. By using integration by parts on each element $T \in \mathcal{T}_{h}$, substituting $\boldsymbol{q}_{h}$ with its numerical flux $\boldsymbol{q}_{h}^{*}$ in the integrals over the elemental boundaries and applying the dG formula (3.3), we obtain

$$
\begin{aligned}
\left(\nabla_{h} \times \boldsymbol{q}_{h}, \boldsymbol{v}_{h}\right)_{\Omega} & =\sum_{T \in \mathcal{T}_{h}}\left(\nabla \times \boldsymbol{q}_{h}, \boldsymbol{v}_{h}\right)_{T}=\sum_{T \in \mathcal{T}_{h}}\left[\left(\boldsymbol{q}_{h}, \nabla \times \boldsymbol{v}_{h}\right)_{T}+\left\langle\boldsymbol{n}_{T} \times \boldsymbol{q}_{h}, \boldsymbol{v}_{h}\right\rangle_{\partial T}\right] \\
& \approx\left(\boldsymbol{q}_{h}, \nabla_{h} \times \boldsymbol{v}_{h}\right)_{\Omega}+\sum_{T \in \mathcal{T}_{h}}\left\langle\boldsymbol{n}_{T} \times \boldsymbol{q}_{h}^{*}, \boldsymbol{v}_{h}\right\rangle_{\partial T} \\
& \left.=\left(\boldsymbol{q}_{h}, \nabla_{h} \times \boldsymbol{v}_{h}\right)_{\Omega}+\sum_{F \in \mathcal{F}_{h}^{I}}\left[\llbracket \llbracket \boldsymbol{q}_{h}^{*} \rrbracket,\left\{\left\{\boldsymbol{v}_{h}\right\}\right\}\right\rangle_{F}-\left\langle\left\{\left\{\boldsymbol{q}_{h}^{*}\right\}\right\}, \llbracket \boldsymbol{v}_{h} \rrbracket\right\rangle_{F}\right]+\sum_{F \in \mathcal{F}_{h}^{B}}\left\langle\boldsymbol{n} \times \boldsymbol{q}_{h}^{*}, \boldsymbol{v}_{h}\right\rangle_{F} .
\end{aligned}
$$

Now we adopt a similar procedure to the second equation (3.1b). Therefore, we consider a test function $\phi_{h} \in$ $\left\{\boldsymbol{f} \in L^{2}\left(\Omega ; \mathbb{C}^{3}\right)|\boldsymbol{f}|_{T} \in C^{0}\left(T ; \mathbb{C}^{3}\right) \cap H^{1}\left(T, \mathbb{C}^{3}\right) \forall T \in \mathcal{T}_{h}\right\}$. Then,

$$
\begin{aligned}
\left(\mu \boldsymbol{q}_{h}, \boldsymbol{\phi}_{h}\right)_{\Omega}= & \left(\nabla_{h} \times \boldsymbol{E}_{h}, \boldsymbol{\phi}_{h}\right)_{\Omega}=\sum_{T \in \mathcal{T}_{h}}\left(\nabla \times \boldsymbol{E}_{h}, \boldsymbol{\phi}_{h}\right)_{T} \\
= & \sum_{T \in \mathcal{T}_{h}}\left[\left(\boldsymbol{E}_{h}, \nabla \times \boldsymbol{\phi}_{h}\right)_{T}+\left\langle\boldsymbol{n}_{T} \times \boldsymbol{E}_{h}, \boldsymbol{\phi}_{h}\right\rangle_{\partial T}\right] \\
\approx & \sum_{T \in \mathcal{T}_{h}}\left[\left(\boldsymbol{E}_{h}, \nabla \times \boldsymbol{\phi}_{h}\right)_{T}+\left\langle\boldsymbol{n}_{T} \times \boldsymbol{E}_{h}^{*}, \boldsymbol{\phi}_{h}\right\rangle_{\partial T}\right] \\
= & \sum_{T \in \mathcal{T}_{h}}\left[\left(\nabla \times \boldsymbol{E}_{h}, \boldsymbol{\phi}_{h}\right)_{T}+\left\langle\boldsymbol{n}_{T} \times\left(\boldsymbol{E}_{h}^{*}-\boldsymbol{E}_{h}\right), \boldsymbol{\phi}_{h}\right\rangle_{\partial T}\right] \\
= & \left(\nabla_{h} \times \boldsymbol{E}_{h}, \boldsymbol{\phi}_{h}\right)_{\Omega}+\sum_{F \in \mathcal{F}_{h}^{I}}\left[\left\langle\llbracket \boldsymbol{E}_{h}^{*}-\boldsymbol{E}_{h} \rrbracket,\left\{\left\{\boldsymbol{\phi}_{h}\right\}\right\}\right\rangle_{F}-\left\langle\left\{\left\{\boldsymbol{E}_{h}^{*}-\boldsymbol{E}_{h}\right\}\right\}, \llbracket \boldsymbol{\phi}_{h} \rrbracket\right\rangle_{F}\right] \\
& +\sum_{F \in \mathcal{F}_{h}^{B}}\left\langle\boldsymbol{n} \times\left(\boldsymbol{E}_{h}^{*}-\boldsymbol{E}_{h}\right), \boldsymbol{\phi}_{h}\right\rangle_{F} .
\end{aligned}
$$

The Sobolev embedding $\mu^{-1} \in W^{1, \infty}(\Omega ; \mathbb{R}) \hookrightarrow C^{0,1}(\bar{\Omega} ; \mathbb{R}) \subset C^{0}(\bar{\Omega} ; \mathbb{R}),\left(c f\right.$. [1], Lem. 4.28), shows that $\mu^{-1} \boldsymbol{\psi}_{h} \in$ $C^{0}\left(T ; \mathbb{C}^{3}\right) \cap H^{1}\left(T ; \mathbb{C}^{3}\right)$ for all $T \in \mathcal{T}_{h}$ and $\boldsymbol{\psi}_{h} \in V_{h, p}$. Hence, the following expressions are all well-defined,

$$
\begin{aligned}
\left(\boldsymbol{q}_{h}, \boldsymbol{\psi}_{h}\right)_{\Omega}= & \left(\mu \boldsymbol{q}_{h}, \mu^{-1} \boldsymbol{\psi}_{h}\right)_{\Omega} \\
= & \left(\nabla_{h} \times \boldsymbol{E}_{h}, \mu^{-1} \boldsymbol{\psi}_{h}\right)_{\Omega}+\sum_{F \in \mathcal{F}_{h}^{I}}\left\langle\llbracket \boldsymbol{E}_{h}^{*}-\boldsymbol{E}_{h} \rrbracket,\left\{\left\{\mu^{-1} \boldsymbol{\psi}_{h}\right\}\right\}\right\rangle_{F} \\
& -\sum_{F \in \mathcal{F}_{h}^{I}}\left\langle\left\{\left\{\boldsymbol{E}_{h}^{*}-\boldsymbol{E}_{h}\right\}\right\}, \llbracket \mu^{-1} \boldsymbol{\psi}_{h} \rrbracket\right\rangle_{F}+\sum_{F \in \mathcal{F}_{h}^{B}}\left\langle\boldsymbol{n} \times\left(\boldsymbol{E}_{h}^{*}-\boldsymbol{E}_{h}\right), \mu^{-1} \boldsymbol{\psi}_{h}\right\rangle_{F},
\end{aligned}
$$


where we used (3.7). Inserting this into (3.6) and the result into (3.5) yields

$$
\begin{aligned}
i \omega \sqrt{\varepsilon_{0}}\left(\boldsymbol{J}_{\boldsymbol{a}}, \boldsymbol{v}_{h}\right)_{\Omega}= & \left(\mu^{-1} \nabla_{h} \times \boldsymbol{E}_{h}, \nabla_{h} \times \boldsymbol{v}_{h}\right)_{\Omega}-\omega^{2}\left(\varepsilon \boldsymbol{E}_{h}, \boldsymbol{v}_{h}\right)_{\Omega}-i \omega\left(\sigma \boldsymbol{E}_{h}, \boldsymbol{v}_{h}\right)_{\Omega} \\
& +\left\langle\llbracket \boldsymbol{E}_{h}^{*}-\boldsymbol{E}_{h} \rrbracket,\left\{\left\{\mu^{-1} \nabla_{h} \times \boldsymbol{v}_{h}\right\}\right\}\right\rangle_{\mathcal{F}_{h}^{I}}-\left\langle\left\{\left\{\boldsymbol{E}_{h}^{*}-\boldsymbol{E}_{h}\right\}\right\}, \llbracket \mu^{-1} \nabla_{h} \times \boldsymbol{v}_{h} \rrbracket\right\rangle_{\mathcal{F}_{h}^{I}} \\
& +\left\langle\boldsymbol{n} \times\left(\boldsymbol{E}_{h}^{*}-\boldsymbol{E}_{h}\right), \mu^{-1} \nabla_{h} \times \boldsymbol{v}_{h}\right\rangle_{\mathcal{F}_{h}^{B}} \\
& +\left\langle\llbracket \boldsymbol{q}_{h}^{*} \rrbracket,\left\{\left\{\boldsymbol{v}_{h}\right\}\right\}\right\rangle_{\mathcal{F}_{h}^{I}}-\left\langle\left\{\left\{\boldsymbol{q}_{h}^{*}\right\}\right\}, \llbracket \boldsymbol{v}_{h} \rrbracket\right\rangle_{\mathcal{F}_{h}^{I}}-\left\langle\boldsymbol{q}_{h}^{*}, \boldsymbol{n} \times \boldsymbol{v}_{h}\right\rangle_{\mathcal{F}_{h}^{B}} .
\end{aligned}
$$

As fluxes $\boldsymbol{E}_{h}^{*}$ and $\boldsymbol{q}_{h}^{*}$ we choose interior penalty fluxes similar to the ones in [22]:

$$
\boldsymbol{E}_{h}^{*}:=\left\{\begin{array}{ll}
\left\{\left\{\boldsymbol{E}_{h}\right\}\right\} & \text { on } F \in \mathcal{F}_{h}^{I}, \\
0 & \text { on } F \in \mathcal{F}_{h}^{\Gamma}, \\
\boldsymbol{E}_{h} & \text { on } F \in \mathcal{F}_{h}^{\Sigma},
\end{array} \quad \boldsymbol{q}_{h}^{*}:= \begin{cases}\left\{\left\{\mu^{-1} \nabla_{h} \times \boldsymbol{E}_{h}\right\}-\tau h_{F}^{-1} \llbracket \boldsymbol{E}_{h} \rrbracket\right. & \text { on } F \in \mathcal{F}_{h}^{I}, \\
\mu^{-1} \nabla_{h} \times \boldsymbol{E}_{h}-\tau h_{F}^{-1}\left(\boldsymbol{n} \times \boldsymbol{E}_{h}\right) & \text { on } F \in \mathcal{F}_{h}^{\Gamma},\end{cases}\right.
$$

where $\tau>0$ is a constant penalty parameter. From the tangential component of the flux $\boldsymbol{q}_{h}^{*}$ on $\Sigma$ we require $\boldsymbol{n} \times \boldsymbol{q}_{h}^{*}:=-\mu_{0}^{-1} \boldsymbol{g}-i \omega \lambda\left(\varepsilon_{0} \mu_{0}^{-1}\right)^{1 / 2}\left(\boldsymbol{E}_{h}\right)_{T}$. Inserting these fluxes into (3.8), using the definition (2.8) of $f$ and observing that $\llbracket\{\{\boldsymbol{u}\}] \rrbracket=0, \llbracket \llbracket \boldsymbol{u} \rrbracket \rrbracket=0,\{\{\{\boldsymbol{u}\}\}\}=\{\{\boldsymbol{u}\}\},\{\{\llbracket \boldsymbol{u} \rrbracket\}\}=\llbracket \boldsymbol{u} \rrbracket$, leads to the following equation

$$
\begin{aligned}
f\left(\boldsymbol{v}_{h} ; \omega\right)= & \left(\mu^{-1} \nabla_{h} \times \boldsymbol{E}_{h}, \nabla_{h} \times \boldsymbol{v}_{h}\right)_{\Omega}-\omega^{2}\left(\varepsilon \boldsymbol{E}_{h}, \boldsymbol{v}_{h}\right)_{\Omega}-i \omega\left(\sigma \boldsymbol{E}_{h}, \boldsymbol{v}_{h}\right)_{\Omega} \\
& -\left\langle\llbracket \boldsymbol{E}_{h} \rrbracket,\left\{\left\{\mu^{-1} \nabla_{h} \times \boldsymbol{v}_{h}\right\}\right\}\right\rangle_{\mathcal{F}_{h}^{I \cup I}}-\left\langle\left\{\left\{\mu^{-1} \nabla_{h} \times \boldsymbol{E}_{h}\right\}\right\}, \llbracket \boldsymbol{v}_{h} \rrbracket\right\rangle_{\mathcal{F}_{h}^{I \cup T}} \\
& +\left\langle\tau \mathrm{h}^{-1} \llbracket \boldsymbol{E}_{h} \rrbracket, \llbracket \boldsymbol{v}_{h} \rrbracket\right\rangle_{\mathcal{F}_{h}^{I \cup \Gamma}}-i \omega \lambda\left(\varepsilon_{0} \mu_{0}^{-1}\right)^{1 / 2}\left\langle\left(\boldsymbol{E}_{h}\right)_{T},\left(\boldsymbol{v}_{h}\right)_{T}\right\rangle_{\Sigma} \\
= & : a_{h}\left(\boldsymbol{E}_{h}, \boldsymbol{v}_{h} ; \omega, \tau\right) .
\end{aligned}
$$

Then, for given $\omega, \tau>0$ the discrete problem reads: Find $\boldsymbol{E}_{h} \in V_{h, p}$ such that

$$
a_{h}\left(\boldsymbol{E}_{h}, \boldsymbol{v}_{h} ; \omega, \tau\right)=f\left(\boldsymbol{v}_{h} ; \omega\right) \quad \forall \boldsymbol{v}_{h} \in V_{h, p} .
$$

Remark 3.1. Since $V_{h, p} \not \subset H(\operatorname{curl}, \Omega)$ and since we use a sesquilinear form $a_{h}$ different from $a_{e}$ in (2.7) (i.e., we have a nonconforming approximation), existence and uniqueness of a solution $\boldsymbol{E}_{h}$ to (3.9) are not obvious.

The above derivation already indicates that the hp-dG discretization is consistent. This is formulated in the next theorem, whose proof is in Appendix A.2.

Theorem 3.2 (Consistency, proof see A.2). The formulation (3.9) is consistent, i.e., if $\boldsymbol{E}$ is the analytical solution to the system (2.1a)-(2.1c), then $\boldsymbol{E}$ satisfies $a_{h}\left(\boldsymbol{E}, \boldsymbol{v}_{h} ; \omega, \tau\right)=f\left(\boldsymbol{v}_{h} ; \omega\right)$ for all $\boldsymbol{v}_{h} \in V_{h, p}$.

\subsection{Continuity and coercivity}

The next step is to show coercivity of $a_{h}$ on $V_{h, p}$ and boundedness of an extension $\widetilde{a}_{h}$ to a vector space containing both, $V_{h, p}$ and $X$, with respect to an energy norm on this space. First, let us define the space $\widetilde{V}_{h, p}$ that relates the spaces $V_{h, p}$ in (3.4) and $X$ in (2.6):

$$
\widetilde{V}_{h, p}:=V_{h, p}+X=\left\{\boldsymbol{v} \in L^{2}\left(\Omega ; \mathbb{C}^{3}\right) \mid \exists \boldsymbol{w}_{h} \in V_{h, p}, \exists \boldsymbol{u} \in X: \boldsymbol{v}=\boldsymbol{w}_{h}+\boldsymbol{u}\right\} .
$$

Note that the sum of $V_{h, p}$ and $X$ is not direct, since $\{0\} \neq V_{h, p} \cap X$. On $\widetilde{V}_{h, p}$ we introduce the dG-norm $\|\cdot\|_{\mathrm{DG}}$ induced by the inner product

$$
\begin{aligned}
\left(\boldsymbol{u}_{h}, \boldsymbol{v}_{h}\right)_{\mathrm{DG}}:= & \left(\boldsymbol{u}_{h}, \boldsymbol{v}_{h}\right)_{\Omega}+\left(\nabla_{h} \times \boldsymbol{u}_{h}, \nabla_{h} \times \boldsymbol{v}_{h}\right)_{\Omega} \\
& +\left\langle\left(\boldsymbol{u}_{h}\right)_{T},\left(\boldsymbol{v}_{h}\right)_{T}\right\rangle_{\Sigma}+\left\langle\mathrm{h}^{-1} \llbracket \boldsymbol{u}_{h} \rrbracket, \llbracket \boldsymbol{v}_{h} \rrbracket\right\rangle_{\mathcal{F}_{h}^{I} \cup \Gamma}, \quad \boldsymbol{u}_{h}, \boldsymbol{v}_{h} \in \widetilde{V}_{h, p},
\end{aligned}
$$


where $\mathrm{h}$ is the function which has been defined in (3.2) and

$$
\|\boldsymbol{w}\|_{\mathcal{F}_{h}^{I \cup \Gamma}}^{2}:=\sum_{F \in \mathcal{F}_{h}^{I} \cup \mathcal{F}_{h}^{\Gamma}}\|\boldsymbol{w}\|_{L^{2}(F)^{3}}^{2}, \quad \boldsymbol{w} \in L^{2}\left(\mathcal{F}_{h}^{I} \cup \mathcal{F}_{h}^{\Gamma} ; \mathbb{C}^{3}\right)
$$

The following discrete version of the trace inequality will be essential for the analysis of the hp-dG scheme.

Lemma 3.3 (Discrete trace inequality). There exists a constant $C_{\mathrm{inv}}>0$, depending only on the shape regularity of the mesh $\mathcal{T}_{h}$ and on the polynomial degree $p$, such that for every $\boldsymbol{v}_{h} \in V_{h, p}$

$$
h_{T}\left\|\boldsymbol{v}_{h}\right\|_{L^{2}(\partial T)^{3}}^{2} \leq C_{\mathrm{inv}}\left\|\boldsymbol{v}_{h}\right\|_{L^{2}(T)^{3}}^{2} \quad \forall T \in \mathcal{T}_{h} .
$$

Proof. According to ([32], Thm. 4), it holds $\left\|\boldsymbol{v}_{h}\right\|_{L^{2}(\partial T)^{3}}^{2} \leq \frac{(p+1)(p+3)}{3} \frac{\text { surface area(T) }}{\text { volume }(T)}\left\|\boldsymbol{v}_{h}\right\|_{L^{2}(T)^{3}}^{2}$ for $\boldsymbol{v}_{h} \in V_{h, p}$ and $T \in \mathcal{T}_{h}$. This implies (3.12) since on shape-regular meshes, there exists a constant $C>0$, independent of the element $T$, such that $\frac{\text { surface area }(T)}{\operatorname{volume}(T)} \leq C h_{T}^{-1}$ for all $T \in \mathcal{T}_{h}$.

Lemma 3.4. For every $\boldsymbol{v}_{h} \in V_{h, p}$ it holds

$$
\left\|\mathrm{h}^{\frac{1}{2}}\left\{\left\{\boldsymbol{v}_{h}\right\}\right\}\right\|_{\mathcal{F}_{h}^{I \cup \Gamma}}^{2} \leq C_{\mathrm{inv}}\left\|\boldsymbol{v}_{h}\right\|_{L^{2}(\Omega)^{3}}^{2},
$$

with $\|\cdot\|_{\mathcal{F}_{h}^{I \cup \Gamma}}$ as in (3.11) and the constant $C_{\mathrm{inv}}$ in (3.12) of Lemma 3.3.

Proof. Let $\boldsymbol{v}_{h} \in V_{h, p}$. Then we can estimate as follows

$$
\begin{aligned}
\left\|\mathrm{h}^{\frac{1}{2}}\left\{\left\{\boldsymbol{v}_{h}\right\}\right\}\right\|_{\mathcal{F}_{h}^{I \cup I}}^{2} & =\sum_{F \in \mathcal{F}_{h}^{I}} \frac{h_{F}}{4}\left\|\left.\boldsymbol{v}_{h}\right|_{T^{L}}+\left.\boldsymbol{v}_{h}\right|_{T^{R}}\right\|_{L^{2}(F)^{3}}^{2}+\sum_{F \in \mathcal{F}_{h}^{I}} h_{F}\left\|\boldsymbol{v}_{h}\right\|_{L^{2}(F)^{3}}^{2} \\
& \leq \frac{1}{2} \sum_{F \in \mathcal{F}_{h}^{I}} h_{F}\left(\left\|\left.\boldsymbol{v}_{h}\right|_{T^{L}}\right\|_{L^{2}(F)^{3}}^{2}+\left\|\left.\boldsymbol{v}_{h}\right|_{T^{R}}\right\|_{L^{2}(F)^{3}}^{2}\right)+\sum_{F \in \mathcal{F}_{h}^{\Gamma}} h_{F}\left\|\boldsymbol{v}_{h}\right\|_{L^{2}(F)^{3}}^{2} \\
& \leq \frac{1}{2} \sum_{F \in \mathcal{F}_{h}^{I}}\left(h_{T^{L}}\left\|\left.\boldsymbol{v}_{h}\right|_{T^{L}}\right\|_{L^{2}(F)^{3}}^{2}+h_{T^{R}}\left\|\left.\boldsymbol{v}_{h}\right|_{T^{R}}\right\|_{L^{2}(F)^{3}}^{2}\right)+\sum_{F \in \mathcal{F}_{h}^{B}} h_{F}\left\|\boldsymbol{v}_{h}\right\|_{L^{2}(F)^{3}}^{2} \\
& \leq \sum_{T \in \mathcal{T}_{h}} \sum_{F \in \mathcal{F}_{h} \cap \partial T} h_{T}\left\|\left.\boldsymbol{v}_{h}\right|_{T}\right\|_{L^{2}(F)^{3}}^{2} \\
& =\sum_{T \in \mathcal{T}_{h}} h_{T}\left\|\left.\boldsymbol{v}_{h}\right|_{T}\right\|_{L^{2}(\partial T)^{3}}^{2} \leq C_{\mathrm{inv}} \sum_{T \in \mathcal{T}_{h}}\left\|\left.\boldsymbol{v}_{h}\right|_{T}\right\|_{L^{2}(T)^{3}}^{2},
\end{aligned}
$$

where we used the discrete trace inequality (3.12) in the last step. This proves the lemma since the last term equals $C_{\mathrm{inv}}\left\|\boldsymbol{v}_{h}\right\|_{L^{2}(\Omega)^{3}}^{2}$.

In order to extend $a_{h}$ to $\widetilde{V}_{h, p} \times \widetilde{V}_{h, p}$, we need the definition of a lifting operator, similar to ([28], Sect. 3.5).

Definition 3.5. Let Assumption 2.1(a) on $\mu$ be satisfied. For $\boldsymbol{u} \in \widetilde{V}_{h, p}$ we define the lifting operator $\mathcal{L}_{\mu}(\boldsymbol{u}) \in$ $V_{h, p}$ via

$$
\left(\mathcal{L}_{\mu}(\boldsymbol{u}), \mu^{-1} \boldsymbol{v}_{h}\right)_{\Omega}=\left\langle\llbracket \boldsymbol{u} \rrbracket,\left\{\left\{\mu^{-1} \boldsymbol{v}_{h}\right\}\right\}\right\rangle_{\mathcal{F}_{h}^{I \cup \Gamma}} \quad \forall \boldsymbol{v}_{h} \in V_{h, p}
$$

Remark 3.6. For $\boldsymbol{u} \in \widetilde{V}_{h, p}$ existence and uniqueness of $\mathcal{L}_{\mu}(\boldsymbol{u}) \in V_{h, p}$ satisfying (3.14) follow from the complexvalued Riesz representation theorem (cf. [25], Thm. 2.30): for $\mu$ as in Assumption 2.1 (a) the form $\left(\cdot, \mu^{-1} \cdot\right)_{\Omega}=$ $\left(\mu^{-1},,\right)_{\Omega}$ is an inner product on $L^{2}\left(\Omega ; \mathbb{C}^{3}\right)$. The space $V_{h, p} \subset L^{2}\left(\Omega ; \mathbb{C}^{3}\right)$ is a closed subspace and for every 
$\boldsymbol{u} \in \widetilde{V}_{h, p}$, the mapping $\ell_{\boldsymbol{u}}^{\mu}\left(\boldsymbol{v}_{h}\right):=\left\langle\left\{\left\{\mu^{-1} \boldsymbol{v}_{h}\right\}\right\}, \llbracket \boldsymbol{u} \rrbracket\right\rangle_{\mathcal{F}_{h}^{I \cup \Gamma}}, \boldsymbol{v}_{h} \in V_{h, p}$, is a bounded linear functional on $V_{h, p}$ : Indeed, inequality (3.13) from above yields

$$
\begin{aligned}
\left|\ell_{\boldsymbol{u}}^{\mu}\left(\boldsymbol{v}_{h}\right)\right| & \leq\left\|\mathrm{h}^{\frac{1}{2}}\left\{\left\{\mu^{-1} \boldsymbol{v}_{h}\right\}\right\}\right\|_{\mathcal{F}_{h}^{I \cup \Gamma}}\left\|\mathrm{h}^{-\frac{1}{2}} \llbracket \boldsymbol{u} \rrbracket\right\|_{\mathcal{F}_{h}^{I \cup \Gamma}} \leq \mu_{-}^{-1}\left\|\mathrm{~h}^{\frac{1}{2}}\left\{\left\{\boldsymbol{v}_{h}\right\}\right\}\right\|_{\mathcal{F}_{h}^{I \cup \Gamma}}\|\boldsymbol{u}\|_{\mathrm{DG}} \\
& \leq\left(\mu_{-}^{-1} \sqrt{C_{\mathrm{inv}}}\|\boldsymbol{u}\|_{\mathrm{DG}}\right)\left\|\boldsymbol{v}_{h}\right\|_{L^{2}(\Omega)^{3}}
\end{aligned}
$$

Now we introduce the extended form $\widetilde{a}_{h}(\cdot, \cdot ; \omega, \tau): \widetilde{V}_{h, p} \times \widetilde{V}_{h, p} \rightarrow \mathbb{C}$ for $\omega, \tau>0$ as

$$
\begin{aligned}
\widetilde{a}_{h}(\boldsymbol{u}, \boldsymbol{v} ; \omega, \tau):= & \left(\mu^{-1} \nabla_{h} \times \boldsymbol{u}, \nabla_{h} \times \boldsymbol{v}\right)_{\Omega}-\omega^{2}(\varepsilon \boldsymbol{u}, \boldsymbol{v})_{\Omega}-i \omega(\sigma \boldsymbol{u}, \boldsymbol{v})_{\Omega} \\
& -\left(\mathcal{L}_{\mu}(\boldsymbol{u}), \mu^{-1} \nabla_{h} \times \boldsymbol{v}\right)_{\Omega}-\left(\mu^{-1} \nabla_{h} \times \boldsymbol{u}, \mathcal{L}_{\mu}(\boldsymbol{v})\right)_{\Omega} \\
& +\left\langle\tau \mathrm{h}^{-1} \llbracket \boldsymbol{u} \rrbracket, \llbracket \boldsymbol{v} \rrbracket\right\rangle_{\mathcal{F}_{h}^{I \cup \Gamma}}-i \omega \lambda\left(\varepsilon_{0} \mu_{0}^{-1}\right)^{1 / 2}\left\langle\boldsymbol{u}_{T}, \boldsymbol{v}_{T}\right\rangle_{\Sigma} .
\end{aligned}
$$

It is obvious, that $\widetilde{a}_{h}(\cdot, \cdot ; \omega, \tau)$ and $a_{h}(\cdot, \cdot ; \omega, \tau)$ coincide on $V_{h, p} \times V_{h, p}$. We will show now that $\widetilde{a}_{h}$ is bounded on $\widetilde{V}_{h, p} \times \widetilde{V}_{h, p}$ w.r.t. the dG-norm $\|\cdot\|_{\text {DG }}$.

Theorem 3.7 (Continuity, proof see A.3). Let Assumption 2.1 be satisfied. Then, the estimate $\left|\widetilde{a}_{h}(\boldsymbol{u}, \boldsymbol{v} ; \omega, \tau)\right| \leq \gamma_{\mathrm{DG}}(\omega)\|\boldsymbol{u}\|_{\mathrm{DG}}\|\boldsymbol{v}\|_{\mathrm{DG}}$ holds for all $\boldsymbol{u}, \boldsymbol{v} \in \widetilde{V}_{h, p}$ and all $\omega, \tau>0$ with

$$
\gamma_{\mathrm{DG}}(\omega):=\max \left\{\mu_{-}^{-1}+\frac{\mu_{+}}{\mu_{-}^{2}} \sqrt{C_{\mathrm{inv}}}, \omega^{2} \varepsilon_{+}+\omega \sigma_{+}, \tau+\frac{\mu_{+}}{\mu_{-}^{2}} \sqrt{C_{\mathrm{inv}}}, \omega \lambda\left(\varepsilon_{0} \mu_{0}^{-1}\right)^{1 / 2}\right\} .
$$

Both, for the proof of Theorem 3.7 and for the error analysis in Section 3.3 below, we need the $\mu^{-1}$ orthogonal $L^{2}$-projection onto $V_{h, p}$.

Definition 3.8. Let Assumption 2.1(a) on $\mu$ be satisfied. For $\boldsymbol{u} \in L^{2}\left(\Omega ; \mathbb{C}^{3}\right)$ the projection $\Pi_{\mu} \boldsymbol{u} \in V_{h, p}$ is defined as the following Riesz representative:

$$
\left(\mu^{-1} \Pi_{\mu} \boldsymbol{u}, \boldsymbol{v}_{h}\right)_{\Omega}=\left(\mu^{-1} \boldsymbol{u}, \boldsymbol{v}_{h}\right)_{\Omega} \quad \forall \boldsymbol{v}_{h} \in V_{h, p}
$$

Note that $\Pi_{\mu}$ satisfies the stability estimate

$$
\left\|\Pi_{\mu} \boldsymbol{u}\right\|_{L^{2}(\Omega)^{3}} \leq \frac{\mu_{+}}{\mu_{-}}\|\boldsymbol{u}\|_{L^{2}(\Omega)^{3}} \quad \forall \boldsymbol{u} \in L^{2}\left(\Omega ; \mathbb{C}^{3}\right)
$$

since it is readily seen that

$$
\mu_{+}^{-1}\left\|\Pi_{\mu} \boldsymbol{u}\right\|_{L^{2}(\Omega)^{3}}^{2} \leq\left(\mu^{-1} \Pi_{\mu} \boldsymbol{u}, \Pi_{\mu} \boldsymbol{u}\right)_{\Omega}=\left(\mu^{-1} \boldsymbol{u}, \Pi_{\mu} \boldsymbol{u}\right)_{\Omega} \leq \mu_{-}^{-1}\|\boldsymbol{u}\|_{L^{2}(\Omega)^{3}}\left\|\Pi_{\mu} \boldsymbol{u}\right\|_{L^{2}(\Omega)^{3}} .
$$

Theorem 3.9 (Coercivity, proof see A.4). Let Assumption 2.1 hold. Then, for all

$$
\tau>\tau^{*}:=\frac{16 C_{\mathrm{inv}} \mu_{+}}{\sigma_{-}^{2} \mu_{-}^{2}}\left(\omega^{2} \varepsilon_{+}^{2}+\sigma_{-}^{2}\right)
$$

and $\omega>0$ it holds that $\left|a_{h}\left(\boldsymbol{v}_{h}, \boldsymbol{v}_{h} ; \omega, \tau\right)\right| \geq \alpha_{\mathrm{DG}}(\omega)\left\|\boldsymbol{v}_{h}\right\|_{\mathrm{DG}}^{2}$ for all $\boldsymbol{v}_{h} \in V_{h, p}$ with

$$
\alpha_{\mathrm{DG}}(\omega):=\min \left\{\frac{\sigma_{-}}{2^{5 / 2} \mu_{+}\left(\omega^{2} \varepsilon_{+}^{2}+\sigma_{-}^{2}\right)^{1 / 2}}, \frac{\omega}{2}\left(\frac{\omega^{2} \varepsilon_{+}^{2} \sigma_{-}^{2}+\sigma_{-}^{4}}{2 \omega^{2} \varepsilon_{+}^{2}+\sigma_{-}^{2}}\right)^{\frac{1}{2}}, \frac{\omega \lambda \sqrt{\varepsilon_{0}}}{\sqrt{2 \mu_{0}}}\right\} .
$$

Corollary 3.10 (Existence and uniqueness of $\boldsymbol{E}_{h}$ ). Let Assumption 2.1 be satisfied. Then, for every frequency $\omega>0$ and $\tau>\tau^{*}$ there exists a unique function $\boldsymbol{E}_{h} \in V_{h, p}$ solving $(3.9)$, i.e., $a_{h}\left(\boldsymbol{E}_{h}, \boldsymbol{v}_{h} ; \omega, \tau\right)=f\left(\boldsymbol{v}_{h} ; \omega\right)$ for all $\boldsymbol{v}_{h} \in V_{h, p}$. 
Proof. Since $V_{h, p}$ is finite dimensional, it is a Hilbert space w.r.t. (3.10). Due to Theorems 3.7 and 3.9, $a_{h}(\cdot, \cdot ; \omega, \tau)$ is bounded and coercive w.r.t. $\|\cdot\|_{\mathrm{DG}}=\sqrt{(\cdot, \cdot)_{\mathrm{DG}}}$ for any $\omega>0$ and $\tau>\tau^{*}$ on $V_{h, p}$ so that the Lax-Milgram theorem yields the assertion.

\subsection{Error in the energy norm}

For the error analysis we collect properties of the $\mu^{-1}$-orthogonal projection $\Pi_{\mu}$ introduced in Definition 3.8. To this end, we introduce the $L^{2}$-orthogonal projection $\Pi_{h}: L^{2}\left(\Omega ; \mathbb{C}^{3}\right) \rightarrow V_{h, p}$ by

$$
\left(\Pi_{h} \boldsymbol{w}, \boldsymbol{v}_{h}\right)_{\Omega}=\left(\boldsymbol{w}, \boldsymbol{v}_{h}\right)_{\Omega} \quad \forall \boldsymbol{v}_{h} \in V_{h, p}, \quad \boldsymbol{w} \in L^{2}\left(\Omega ; \mathbb{C}^{3}\right) .
$$

Furthermore, we define $\Delta_{\boldsymbol{a}}$ for a node $\boldsymbol{a}$ in the mesh $\mathcal{T}_{h}$ as the set of all tetrahedra sharing this vertex.

Lemma 3.11. There exists a constant $C>0$, independent of $T$ and $h_{T}$, such that for any $\boldsymbol{v} \in L^{2}\left(\Omega ; \mathbb{C}^{3}\right)$ with $\left.\boldsymbol{v}\right|_{\Delta_{a}} \in H^{t}\left(\Delta_{\boldsymbol{a}} ; \mathbb{C}^{3}\right), t>\frac{1}{2}$, any $T \in \mathcal{T}_{h}$ and any vertex $\boldsymbol{a}$ of $T$ the projection $\Pi_{h}$ in (3.18) satisfies

$$
\left\|\boldsymbol{v}-\Pi_{h} \boldsymbol{v}\right\|_{L^{2}(T)^{3}}^{2}+h_{T}\left\|\boldsymbol{v}-\Pi_{h} \boldsymbol{v}\right\|_{L^{2}(\partial T)^{3}}^{2} \leq C h_{T}^{2 \min \{t, p+1\}}\|\boldsymbol{v}\|_{H^{t}\left(\Delta_{a}\right)^{3}}^{2} .
$$

Proof. In two dimensions, this theorem follows from the properties of the interpolation operator in ([12], Thm. 1) or ([6], Thm. 2.1 and Rem. 4). As mentioned in ([18], Sect. A.3), the proof can be directly adopted to the case of higher dimensions.

The estimate (3.19) for the $L^{2}$-orthogonal projection $\Pi_{h}$ on $V_{h, p}$ provides us now also with an error bound for the $\mu^{-1}$-orthogonal projection $\Pi_{\mu}$ in Definition 3.8 .

Lemma 3.12. There exists a constant $C>0$ depending only on $p, \mu$ and the shape regularity of the mesh such that for any $\boldsymbol{v} \in L^{2}\left(\Omega ; \mathbb{C}^{3}\right)$ with $\left.\boldsymbol{v}\right|_{\Delta_{a}} \in H^{t}\left(\Delta_{\boldsymbol{a}} ; \mathbb{C}^{3}\right), t>\frac{1}{2}$, any $T \in \mathcal{T}_{h}$ and any vertex $\boldsymbol{a}$ of $T$ the projection $\Pi_{\mu}$ in (3.15) satisfies

$$
\left\|\boldsymbol{v}-\Pi_{\mu} \boldsymbol{v}\right\|_{L^{2}(T)^{3}}^{2}+h_{T}\left\|\boldsymbol{v}-\Pi_{\mu} \boldsymbol{v}\right\|_{L^{2}(\partial T)^{3}}^{2} \leq C h_{T}^{2 \min \{t, p+1\}}\|\boldsymbol{v}\|_{H^{t}\left(\Delta_{a}\right)^{3}}^{2} .
$$

Proof. Let $T \in \mathcal{T}_{h}, \boldsymbol{a}$ be a vertex of $T$ and $\boldsymbol{v} \in H^{t}\left(\Delta_{\boldsymbol{a}} ; \mathbb{C}^{3}\right)$ for some $t>\frac{1}{2}$. Then,

$$
\begin{aligned}
\mu_{+}^{-1}\left\|\Pi_{h} \boldsymbol{v}-\Pi_{\mu} \boldsymbol{v}\right\|_{L^{2}(T)^{3}}^{2} & \leq\left(\mu^{-1}\left(\Pi_{h} \boldsymbol{v}-\Pi_{\mu} \boldsymbol{v}\right), \Pi_{h} \boldsymbol{v}-\Pi_{\mu} \boldsymbol{v}\right)_{T} \\
& =\left(\mu^{-1} \Pi_{h} \boldsymbol{v}, \Pi_{h} \boldsymbol{v}-\Pi_{\mu} \boldsymbol{v}\right)_{T}-\left(\mu^{-1} \boldsymbol{v}, \Pi_{h} \boldsymbol{v}-\Pi_{\mu} \boldsymbol{v}\right)_{T} \\
& =\left(\mu^{-1}\left(\Pi_{h} \boldsymbol{v}-\boldsymbol{v}\right), \Pi_{h} \boldsymbol{v}-\Pi_{\mu} \boldsymbol{v}\right)_{T} \leq \mu_{-}^{-1}\left\|\Pi_{h} \boldsymbol{v}-\boldsymbol{v}\right\|_{L^{2}(T)^{3}}\left\|\Pi_{h} \boldsymbol{v}-\Pi_{\mu} \boldsymbol{v}\right\|_{L^{2}(T)^{3}}
\end{aligned}
$$

and, therefore,

$$
\left\|\Pi_{h} \boldsymbol{v}-\Pi_{\mu} \boldsymbol{v}\right\|_{L^{2}(T)^{3}} \leq \frac{\mu_{+}}{\mu_{-}}\left\|\Pi_{h} \boldsymbol{v}-\boldsymbol{v}\right\|_{L^{2}(T)^{3}},
$$

as well as

$$
\begin{aligned}
\left\|\boldsymbol{v}-\Pi_{\mu} \boldsymbol{v}\right\|_{L^{2}(T)^{3}} & \leq\left\|\boldsymbol{v}-\Pi_{h} \boldsymbol{v}\right\|_{L^{2}(T)^{3}}+\left\|\Pi_{h} \boldsymbol{v}-\Pi_{\mu} \boldsymbol{v}\right\|_{L^{2}(T)^{3}} \\
& \leq\left(1+\frac{\mu_{+}}{\mu_{-}}\right)\left\|\boldsymbol{v}-\Pi_{h} \boldsymbol{v}\right\|_{L^{2}(T)^{3}} \leq\left(1+\frac{\mu_{+}}{\mu_{-}}\right) C h_{T}^{\min \{t, p+1\}}\|\boldsymbol{v}\|_{H^{t}\left(\Delta_{a}\right)^{3}}
\end{aligned}
$$


for any corner $\boldsymbol{a}$ of $T \in \mathcal{T}_{h}$ by Lemma 3.11. For the $L^{2}$-norm on $\partial T$ we may use the discrete trace inequality (3.12), (3.19) for $\Pi_{h}$ and (3.21). Thus,

$$
\begin{aligned}
h_{T}\left\|\boldsymbol{v}-\Pi_{\mu} \boldsymbol{v}\right\|_{L^{2}(\partial T)^{3}}^{2} & \leq h_{T}\left(\left\|\boldsymbol{v}-\Pi_{h} \boldsymbol{v}\right\|_{L^{2}(\partial T)^{3}}+\left\|\Pi_{h} \boldsymbol{v}-\Pi_{\mu} \boldsymbol{v}\right\|_{L^{2}(\partial T)^{3}}\right)^{2} \\
& \leq 2 h_{T}\left\|\boldsymbol{v}-\Pi_{h} \boldsymbol{v}\right\|_{L^{2}(\partial T)^{3}}^{2}+2 h_{T}\left\|\Pi_{h} \boldsymbol{v}-\Pi_{\mu} \boldsymbol{v}\right\|_{L^{2}(\partial T)^{3}}^{2} \\
& \leq 2 h_{T}\left\|\boldsymbol{v}-\Pi_{h} \boldsymbol{v}\right\|_{L^{2}(\partial T)^{3}}^{2}+2 C_{\mathrm{inv}}\left\|\Pi_{h} \boldsymbol{v}-\Pi_{\mu} \boldsymbol{v}\right\|_{L^{2}(T)^{3}}^{2} \\
& \leq 2 \max \left\{1, C_{\mathrm{inv}} \mu_{+}^{2} \mu_{-}^{-2}\right\}\left(h_{T}\left\|\boldsymbol{v}-\Pi_{h} \boldsymbol{v}\right\|_{L^{2}(\partial T)^{3}}^{2}+\left\|\boldsymbol{v}-\Pi_{h} \boldsymbol{v}\right\|_{L^{2}(T)^{3}}^{2}\right) \\
& \leq C h_{T}^{2 \min \{t, p+1\}}\|\boldsymbol{v}\|_{H^{t}\left(\Delta_{a}\right)^{3}}^{2},
\end{aligned}
$$

so that the lemma is proven.

For $\omega, \tau>0$ we define the residual for $\boldsymbol{v}_{h} \in V_{h, p}$ by

$$
r_{h}\left(\boldsymbol{v}_{h} ; \omega, \tau\right):=\widetilde{a}_{h}\left(\boldsymbol{E}, \boldsymbol{v}_{h} ; \omega, \tau\right)-f\left(\boldsymbol{v}_{h} ; \omega\right)=\widetilde{a}_{h}\left(\boldsymbol{E}-\boldsymbol{E}_{h}, \boldsymbol{v}_{h} ; \omega, \tau\right),
$$

where $\boldsymbol{E}$ denotes the exact solution of (2.1a)-(2.1c) and $\boldsymbol{E}_{h}$ the dG approximation as a solution of (3.9). We are able to estimate the absolute value of the residual $r_{h}$ as follows.

Proposition 3.13. Let Assumption 2.1 be satisfied, and $\boldsymbol{E}$ be the unique exact solution to (2.1a)-(2.1c) for a frequency $\omega>0$ with $\nabla \times \boldsymbol{E} \in H^{t}\left(\Omega ; \mathbb{C}^{3}\right)$ for some $t>\frac{1}{2}$. Then, for $\tau>0$, the residual can be expressed as

$$
r_{h}\left(\boldsymbol{v}_{h} ; \omega, \tau\right)=\left\langle\left\{\left\{\mu^{-1}\left(\nabla \times \boldsymbol{E}-\Pi_{\mu}(\nabla \times \boldsymbol{E})\right)\right\}\right\}, \llbracket \boldsymbol{v}_{h} \rrbracket\right\rangle_{\mathcal{F}_{h}^{I \cup \Gamma}} \quad \forall \boldsymbol{v}_{h} \in V_{h, p} .
$$

In addition, there exists a constant $C>0$ depending only on $p, \mu$ and the shape regularity of the mesh, such that

$$
\left|r_{h}\left(\boldsymbol{v}_{h} ; \omega, \tau\right)\right| \leq C h^{\min \{t, p+1\}}\left\|\boldsymbol{v}_{h}\right\|_{\mathrm{DG}}\|\nabla \times \boldsymbol{E}\|_{H^{t}(\Omega)^{3}} \quad \forall \boldsymbol{v}_{h} \in V_{h, p} .
$$

Proof. In order to derive representation (3.22), let $\omega, \tau>0$, and $\boldsymbol{v}_{h} \in V_{h, p}$. Then,

$$
\begin{aligned}
& r_{h}\left(\boldsymbol{v}_{h} ; \omega, \tau\right)=\widetilde{a}_{h}\left(\boldsymbol{E}, \boldsymbol{v}_{h} ; \omega, \tau\right)-f\left(\boldsymbol{v}_{h} ; \omega\right) \\
&=\left(\mu^{-1} \nabla \times \boldsymbol{E}, \nabla_{h} \times \boldsymbol{v}_{h}\right)_{\Omega}-\omega^{2}\left(\varepsilon \boldsymbol{E}, \boldsymbol{v}_{h}\right)_{\Omega}-i \omega\left(\sigma \boldsymbol{E}, \boldsymbol{v}_{h}\right)_{\Omega} \\
&-\left(\mu^{-1} \nabla \times \boldsymbol{E}, \mathcal{L}_{\mu}\left(\boldsymbol{v}_{h}\right)\right)_{\Omega}-i \omega \lambda\left(\varepsilon_{0} \mu_{0}^{-1}\right)^{1 / 2}\left\langle\boldsymbol{E}_{T},\left(\boldsymbol{v}_{h}\right)_{T}\right\rangle_{\Sigma} \\
&-\left(\mathcal{L}_{\mu}(\boldsymbol{E}), \mu^{-1} \nabla_{h} \times \boldsymbol{v}_{h}\right)_{\Omega}+\left\langle\tau \mathrm{h}^{-1} \llbracket \boldsymbol{E} \rrbracket, \llbracket \boldsymbol{v}_{h} \rrbracket\right\rangle_{\mathcal{F}_{h}^{I} \cup \Gamma}-f\left(\boldsymbol{v}_{h} ; \omega\right) .
\end{aligned}
$$

The first two expressions in the last line vanish since $\llbracket \boldsymbol{E} \rrbracket=0$ on $\mathcal{F}_{h}^{I \cup \Gamma}$ and, hence, $\mathcal{L}_{\mu}(\boldsymbol{E})=0$. Applying integration by parts to the integral $\left(\mu^{-1} \nabla \times \boldsymbol{E}, \nabla \times \boldsymbol{v}_{h}\right)_{T}$ on every element $T \in \mathcal{T}_{h}$ and afterwards the dG formula (3.3) yields

$$
\begin{aligned}
& r_{h}\left(\boldsymbol{v}_{h} ; \omega, \tau\right)=\left(\nabla \times\left(\mu^{-1} \nabla \times \boldsymbol{E}\right), \boldsymbol{v}_{h}\right)_{\Omega}-\left(\left(\omega^{2} \varepsilon+i \omega \sigma\right) \boldsymbol{E}, \boldsymbol{v}_{h}\right)_{\Omega}-\left(\mu^{-1} \Pi_{\mu}(\nabla \times \boldsymbol{E}), \mathcal{L}_{\mu}\left(\boldsymbol{v}_{h}\right)\right)_{\Omega} \\
& -\sum_{T \in \mathcal{T}_{h}}\left\langle\boldsymbol{n}_{T} \times\left(\mu^{-1} \nabla \times \boldsymbol{E}\right), \boldsymbol{v}_{h}\right\rangle_{\partial T}-i \omega \lambda\left(\varepsilon_{0} \mu_{0}^{-1}\right)^{1 / 2}\left\langle\boldsymbol{E}_{T},\left(\boldsymbol{v}_{h}\right)_{T}\right\rangle_{\Sigma}-\mu_{0}^{-1}\left\langle\boldsymbol{g},\left(\boldsymbol{v}_{h}\right)_{T}\right\rangle_{\Sigma} \\
& -i \omega \sqrt{\varepsilon_{0}}\left(\boldsymbol{J}_{\boldsymbol{a}}, \boldsymbol{v}_{h}\right)_{\Omega} \\
& =-\left\langle\llbracket \mu^{-1} \nabla \times \boldsymbol{E} \rrbracket,\left\{\left\{\boldsymbol{v}_{h}\right\}\right\}\right\rangle_{\mathcal{F}_{h}^{I}}+\left\langle\left\{\left\{\mu^{-1} \nabla \times \boldsymbol{E}\right\}\right\}, \llbracket \boldsymbol{v}_{h} \rrbracket\right\rangle_{\mathcal{F}_{h}^{I}}+\left\langle\mu^{-1} \nabla \times \boldsymbol{E}, \boldsymbol{n} \times \boldsymbol{v}_{h}\right\rangle_{\mathcal{F}_{h}^{\Gamma}} \\
& -\left\langle\boldsymbol{n} \times\left(\mu^{-1} \nabla \times \boldsymbol{E}\right), \boldsymbol{v}_{h}\right\rangle_{\mathcal{F}_{h}^{\Sigma}}-\left\langle\left\{\left\{\mu^{-1} \Pi_{\mu}(\nabla \times \boldsymbol{E})\right\}, \llbracket \boldsymbol{v}_{h} \rrbracket\right\rangle_{\mathcal{F}_{h}^{I \cup \Gamma}}-\mu_{0}^{-1}\left\langle\boldsymbol{g},\left(\boldsymbol{v}_{h}\right)_{T}\right\rangle_{\Sigma}\right. \\
& -i \omega \lambda\left(\varepsilon_{0} \mu_{0}^{-1}\right)^{1 / 2}\left\langle\boldsymbol{E}_{T},\left(\boldsymbol{v}_{h}\right)_{T}\right\rangle_{\Sigma} \\
& =\left\langle\left\{\left\{\mu^{-1} \nabla \times \boldsymbol{E}-\mu^{-1} \Pi_{\mu}(\nabla \times \boldsymbol{E})\right\}\right\}, \llbracket \boldsymbol{v}_{h} \rrbracket\right\rangle_{\mathcal{F}_{h}^{I \cup \Gamma}}-\left\langle\boldsymbol{n} \times\left(\mu^{-1} \nabla \times \boldsymbol{E}\right)\right. \\
& \left.+i \omega \lambda\left(\varepsilon_{0} \mu_{0}^{-1}\right)^{1 / 2} \boldsymbol{E}_{T}+\mu_{0}^{-1} \boldsymbol{g},\left(\boldsymbol{v}_{h}\right)_{T}\right\rangle_{\Sigma}=\left\langle\left\{\left\{\mu^{-1}\left(\nabla \times \boldsymbol{E}-\Pi_{\mu}(\nabla \times \boldsymbol{E})\right)\right\}\right\}, \llbracket \boldsymbol{v}_{h} \rrbracket\right\rangle_{\mathcal{F}_{h}^{I \cup T}},
\end{aligned}
$$


since $\llbracket \mu^{-1} \nabla \times \boldsymbol{E} \rrbracket=0$ on $\mathcal{F}_{h}^{I},\left\langle\boldsymbol{n} \times\left(\mu^{-1} \nabla \times \boldsymbol{E}\right), \boldsymbol{v}_{h}\right\rangle_{\mathcal{F}_{h}}=\left\langle\boldsymbol{n} \times\left(\mu^{-1} \nabla \times \boldsymbol{E}\right),\left(\boldsymbol{v}_{h}\right)_{T}\right\rangle_{\Sigma}$ and $\boldsymbol{E}$ satisfies $(2.1 \mathrm{c})$. Using this representation, we can estimate as follows

$$
\begin{aligned}
& \left|r_{h}\left(\boldsymbol{v}_{h} ; \omega, \tau\right)\right|=\left|\left\langle\left\{\left\{\mu^{-1}\left(\nabla \times \boldsymbol{E}-\Pi_{\mu}(\nabla \times \boldsymbol{E})\right)\right\}\right\}, \llbracket \boldsymbol{v}_{h} \rrbracket\right\rangle_{\mathcal{F}_{h}^{I \cup T}}\right| \\
& \leq\left\|\mathrm{h}^{-\frac{1}{2}} \llbracket \boldsymbol{v}_{h} \rrbracket\right\|_{\mathcal{F}_{h}^{I \cup \Gamma}}\left\|\mathrm{h}^{\frac{1}{2}}\left\{\left\{\mu^{-1}\left(\nabla \times \boldsymbol{E}-\Pi_{\mu}(\nabla \times \boldsymbol{E})\right)\right\}\right\}\right\|_{\mathcal{F}_{h}^{I \cup \Gamma}} \\
& \leq\left\|\boldsymbol{v}_{h}\right\|_{\mathrm{DG}}\left(\sum_{F \in \mathcal{F}_{h}} h_{F} \|\left\{\left\{\mu^{-1}\left(\nabla \times \boldsymbol{E}-\Pi_{\mu}(\nabla \times \boldsymbol{E})\right)\right\} \|_{L^{2}(F)^{3}}^{2}\right)^{\frac{1}{2}}\right. \\
& \leq\left\|\boldsymbol{v}_{h}\right\|_{\mathrm{DG}}\left(\sum_{T \in \mathcal{T}_{h}} h_{T}\left\|\mu^{-1}\left(\nabla \times \boldsymbol{E}-\Pi_{\mu}(\nabla \times \boldsymbol{E})\right)\right\|_{L^{2}(\partial T)^{3}}^{2}\right)^{\frac{1}{2}} \\
& \leq \mu_{-}^{-1}\left\|\boldsymbol{v}_{h}\right\|_{\mathrm{DG}}\left(\sum_{T \in \mathcal{T}_{h}} h_{T}\left\|\left(\nabla \times \boldsymbol{E}-\Pi_{\mu}(\nabla \times \boldsymbol{E})\right)\right\|_{L^{2}(\partial T)^{3}}^{2}\right)^{\frac{1}{2}} \\
& \leq C h^{\min \{t, p+1\}}\left\|\boldsymbol{v}_{h}\right\|_{\mathrm{DG}}\|\nabla \times \boldsymbol{E}\|_{H^{t}(\Omega)^{3}},
\end{aligned}
$$

where we used estimate (3.20) for $\Pi_{\mu}$ in the last step.

Next, we state an error estimate for the Nédélec interpolant w.r.t. $\|\cdot\|_{X}$.

Lemma 3.14 (Nédélec interpolant). Let $\boldsymbol{v} \in H^{t}\left(\Omega ; \mathbb{C}^{3}\right)$ with $\nabla \times \boldsymbol{v} \in H^{t}\left(\Omega ; \mathbb{C}^{3}\right)$ and $\boldsymbol{v}_{T} \in H_{\|}^{t}(\Sigma)^{3} \cap$ $H\left(\operatorname{curl}_{\Sigma}, \Sigma\right)$ for some $t>\frac{1}{2}$, where

$$
H_{\|}^{t}(\Sigma)^{3}:=\left\{\boldsymbol{w} \in L_{t}^{2}\left(\Sigma ; \mathbb{C}^{3}\right) \mid \exists \boldsymbol{\xi} \in H^{t+\frac{1}{2}}\left(\Omega ; \mathbb{C}^{3}\right): \boldsymbol{w}=\gamma_{T}(\boldsymbol{\xi}):=\left(\boldsymbol{n} \times\left.\boldsymbol{\xi}\right|_{\Sigma}\right) \times \boldsymbol{n}\right\}
$$

and $H\left(\operatorname{curl}_{\Sigma}, \Sigma\right):=\left\{\boldsymbol{w} \in L_{t}^{2}\left(\Sigma ; \mathbb{C}^{3}\right) \mid \nabla_{\Sigma} \times \boldsymbol{w} \in L^{2}(\Sigma ; \mathbb{C})\right\}$ with the surface curl operator $\nabla_{\Sigma} \times$ on the surface $\Sigma$, (cf. [8], Prop. 3.6). Then, there exists a function $\Pi_{N} \boldsymbol{v} \in V_{h, p} \cap X$ satisfying

$$
\begin{aligned}
\left\|\boldsymbol{v}-\Pi_{N} \boldsymbol{v}\right\|_{X} & =\left(\left\|\boldsymbol{v}-\Pi_{N} \boldsymbol{v}\right\|_{H(\operatorname{curl}, \Omega)}^{2}+\left\|\left(\boldsymbol{v}-\Pi_{N} \boldsymbol{v}\right)_{T}\right\|_{L^{2}(\Sigma)^{3}}^{2}\right)^{\frac{1}{2}} \\
& \leq C h^{\min \{t, p\}}\left(\|\boldsymbol{v}\|_{H^{t}(\Omega)^{3}}+\|\nabla \times \boldsymbol{v}\|_{H^{t}(\Omega)^{3}}+\left\|\boldsymbol{v}_{T}\right\|_{H_{\|}^{t}(\Sigma)^{3}}+\left\|\nabla_{\Sigma} \times\left(\boldsymbol{v}_{T}\right)\right\|_{L^{2}(\Sigma)}\right),
\end{aligned}
$$

where $\|\boldsymbol{w}\|_{H_{\|}^{t}(\Sigma)^{3}}:=\inf _{\boldsymbol{\xi} \in H^{t+\frac{1}{2}(\Omega)^{3}}}\left\{\|\boldsymbol{\xi}\|_{H^{t+\frac{1}{2}(\Omega)^{3}}} \mid \gamma_{T}(\boldsymbol{\xi})=\boldsymbol{w}\right\}$ and $C>0$ is a constant depending only on $p \in \mathbb{N}$ and the shape regularity of the mesh.

Proof. It is a well-known result $(c f .[2,26])$ that the interpolation operator $\Pi_{N}: H(\operatorname{curl}, \Omega) \rightarrow N_{h}^{p}(\Omega)$ on the finite element space $N_{h}^{p}(\Omega)$ generated by the Nédélec elements of first kind and $p$ th degree satisfies

$$
\left\|\boldsymbol{v}-\Pi_{N} \boldsymbol{v}\right\|_{H(\operatorname{curl}, \Omega)} \leq C h^{\min \{t, p\}}\left(\|\boldsymbol{v}\|_{H^{t}(\Omega)^{3}}+\|\nabla \times \boldsymbol{v}\|_{H^{t}(\Omega)^{3}}\right)
$$

for some constant $C>0$, independent of the mesh size $h$, if $\boldsymbol{v} \in H^{t}\left(\Omega, \mathbb{C}^{3}\right)$ and $\nabla \times \boldsymbol{v} \in H^{t}\left(\Omega, \mathbb{C}^{3}\right)$ for some $t>\frac{1}{2}$.

It remains to investigate $\left\|\left(\boldsymbol{v}-\Pi_{N} \boldsymbol{v}\right)_{T}\right\|_{L^{2}(\Sigma)^{3}}$. As argued in [17], Section 5.1, the result of ([9], Lem. 15) yields an error estimate for the interpolation operator $\Pi_{N}^{\Sigma}$ on the finite element space $N_{h}^{p}(\Sigma) \subset H\left(\operatorname{curl}_{\Sigma}, \Sigma\right)$ defined by

$$
N_{h}^{p}(\Sigma):=\gamma_{T}\left(N_{h}^{p}(\Omega)\right)=\left\{\boldsymbol{g} \in L_{t}^{2}\left(\Sigma ; \mathbb{C}^{3}\right): \exists \boldsymbol{\xi} \in N_{h}^{p}(\Omega) \text { with } \boldsymbol{g}=\gamma_{T}(\boldsymbol{\xi})\right\} .
$$

To be specific, for every $\boldsymbol{w} \in H_{\|}^{t}(\Sigma)^{3} \cap H\left(\operatorname{curl}_{\Sigma}, \Sigma\right), t>\frac{1}{2}$, it holds

$$
\left\|\boldsymbol{w}-\Pi_{N}^{\Sigma} \boldsymbol{w}\right\|_{L^{2}(\Sigma)^{3}} \leq C h^{\min \{t, p+1\}}\left(\|\boldsymbol{w}\|_{H_{\|}^{t}(\Sigma)^{3}}+\left\|\nabla_{\Sigma} \times \boldsymbol{w}\right\|_{L^{2}(\Sigma)}\right)
$$


for some constant $C>0$, independent of $h$. Finally, the commuting diagram property $\gamma_{T} \Pi_{N}=\Pi_{N}^{\Sigma} \gamma_{T}(c f$. [17], Sect. 5.1) implies that

$$
\left\|\left(\boldsymbol{v}-\Pi_{N}\right)_{T}\right\|_{L^{2}(\Sigma)^{3}} \leq C h^{\min \{t, p+1\}}\left(\left\|\boldsymbol{v}_{T}\right\|_{H_{\|}^{t}(\Sigma)^{3}}+\left\|\nabla_{\Sigma} \times\left(\boldsymbol{v}_{T}\right)\right\|_{L^{2}(\Sigma)}\right),
$$

if $\boldsymbol{v}_{T} \in H_{\|}^{t}(\Sigma)^{3} \cap H\left(\operatorname{curl}_{\Sigma}, \Sigma\right)$, and the assertion is proven.

Proposition 3.15 (Partition of the error). Let Assumption 2.1 be satisfied, and $\boldsymbol{E}$ solve (2.1a)-(2.1c) for $\omega>0$. If $\boldsymbol{E}_{h}$ is the $d G$ approximation in (3.9) for $\tau>\tau^{*}$, then it holds

$$
\left\|\boldsymbol{E}-\boldsymbol{E}_{h}\right\|_{\mathrm{DG}} \leq K\left(\inf _{\boldsymbol{v}_{h} \in V_{h, p}}\left\|\boldsymbol{E}-\boldsymbol{v}_{h}\right\|_{\mathrm{DG}}+\sup _{\boldsymbol{w}_{h} \in V_{h, p} \backslash\{0\}} \frac{\left|r_{h}\left(\boldsymbol{w}_{h} ; \omega, \tau\right)\right|}{\left\|\boldsymbol{w}_{h}\right\|_{\mathrm{DG}}}\right)
$$

with

$$
K=K(\omega):=\frac{\max \left\{1, \gamma_{\mathrm{DG}}(\omega)+\alpha_{\mathrm{DG}}(\omega)\right\}}{\alpha_{\mathrm{DG}}(\omega)} .
$$

Proof. Let $\boldsymbol{v}_{h} \in V_{h, p}$. Then, $\left\|\boldsymbol{E}-\boldsymbol{E}_{h}\right\|_{\mathrm{DG}} \leq\left\|\boldsymbol{E}-\boldsymbol{v}_{h}\right\|_{\mathrm{DG}}+\left\|\boldsymbol{v}_{h}-\boldsymbol{E}_{h}\right\|_{\mathrm{DG}}$, and using coercivity and continuity of $a_{h}$ and $\widetilde{a}_{h}$, respectively,

$$
\begin{aligned}
\alpha_{\mathrm{DG}}\left\|\boldsymbol{v}_{h}-\boldsymbol{E}_{h}\right\|_{\mathrm{DG}}^{2} & \leq\left|a_{h}\left(\boldsymbol{v}_{h}-\boldsymbol{E}_{h}, \boldsymbol{v}_{h}-\boldsymbol{E}_{h} ; \omega, \tau\right)\right|=\left|\widetilde{a}_{h}\left(\boldsymbol{v}_{h}-\boldsymbol{E}_{h}, \boldsymbol{v}_{h}-\boldsymbol{E}_{h} ; \omega, \tau\right)\right| \\
& \leq\left|\widetilde{a}_{h}\left(\boldsymbol{v}_{h}-\boldsymbol{E}, \boldsymbol{v}_{h}-\boldsymbol{E}_{h} ; \omega, \tau\right)\right|+\left|\widetilde{a}_{h}\left(\boldsymbol{E}-\boldsymbol{E}_{h}, \boldsymbol{v}_{h}-\boldsymbol{E}_{h} ; \omega, \tau\right)\right| \\
& \leq \gamma_{\mathrm{DG}}\left\|\boldsymbol{v}_{h}-\boldsymbol{E}\right\|_{\mathrm{DG}}\left\|\boldsymbol{v}_{h}-\boldsymbol{E}_{h}\right\|_{\mathrm{DG}}+\left|r_{h}\left(\boldsymbol{v}_{h}-\boldsymbol{E}_{h} ; \omega, \tau\right)\right|
\end{aligned}
$$

which implies

$$
\begin{aligned}
\left\|\boldsymbol{E}-\boldsymbol{E}_{h}\right\|_{\mathrm{DG}} & \leq\left(1+\frac{\gamma_{\mathrm{DG}}}{\alpha_{\mathrm{DG}}}\right)\left\|\boldsymbol{v}_{h}-\boldsymbol{E}\right\|_{\mathrm{DG}}+\frac{1}{\alpha_{\mathrm{DG}}} \frac{\left|r_{h}\left(\boldsymbol{v}_{h}-\boldsymbol{E}_{h} ; \omega, \tau\right)\right|}{\left\|\boldsymbol{v}_{h}-\boldsymbol{E}_{h}\right\|_{\mathrm{DG}}} \\
& \leq \frac{\max \left\{1, \gamma_{\mathrm{DG}}+\alpha_{\mathrm{DG}}\right\}}{\alpha_{\mathrm{DG}}}\left(\left\|\boldsymbol{v}_{h}-\boldsymbol{E}\right\|_{\mathrm{DG}}+\sup _{\boldsymbol{w}_{h} \in V_{h, p}} \frac{\left|r_{h}\left(\boldsymbol{w}_{h} ; \omega, \tau\right)\right|}{\left\|\boldsymbol{w}_{h}\right\|_{\mathrm{DG}}}\right) .
\end{aligned}
$$

The assertion follows now by taking the infimum over $\boldsymbol{v}_{h}$ in $V_{h, p}$.

Theorem 3.16 (Error in the energy norm). Let Assumption 2.1 be satisfied and assume that the solution $\boldsymbol{E}$ of (2.1a)- (2.1c) for $\omega>0$ satisfies $\boldsymbol{E} \in H^{t}\left(\Omega ; \mathbb{C}^{3}\right)$ with $\nabla \times \boldsymbol{E} \in H^{t}\left(\Omega ; \mathbb{C}^{3}\right)$ and $\boldsymbol{E}_{T} \in H_{\|}^{t}(\Sigma)^{3} \cap H\left(\operatorname{curl}_{\Sigma}, \Sigma\right)$ on $\Sigma$ for some $t>\frac{1}{2}$. Let $\boldsymbol{E}_{h} \in V_{h, p}$ be the $d G$ approximation solving (3.9) for $\tau>\tau^{*}$ and define $K$ as in (3.23). Then, there exists a constant, independent of $h$ and $\omega$, such that

$$
\left\|\boldsymbol{E}-\boldsymbol{E}_{h}\right\|_{\mathrm{DG}} \leq C K h^{\min \{t, p\}}\left(\|\boldsymbol{E}\|_{H^{t}(\Omega)^{3}}+\|\nabla \times \boldsymbol{E}\|_{H^{t}(\Omega)^{3}}+\left\|\boldsymbol{E}_{T}\right\|_{H_{\|}^{t}(\Sigma)^{3}}+\left\|\nabla_{\Sigma} \times\left(\boldsymbol{E}_{T}\right)\right\|_{L^{2}(\Sigma)}\right) .
$$

Proof. The result follows from Propositions 3.13, 3.15 and Lemma 3.14:

$$
\begin{aligned}
\left\|\boldsymbol{E}-\boldsymbol{E}_{h}\right\|_{\mathrm{DG}} & \leq K\left(\inf _{\boldsymbol{v}_{h} \in V_{h, p}}\left\|\boldsymbol{E}-\boldsymbol{v}_{h}\right\|_{\mathrm{DG}}+\sup _{\boldsymbol{w}_{h} \in V_{h, p}} \frac{\left|r_{h}\left(\boldsymbol{w}_{h} ; \omega, \tau\right)\right|}{\left\|\boldsymbol{w}_{h}\right\|_{\mathrm{DG}}}\right) \\
& \leq C K\left(\inf _{\boldsymbol{v}_{h} \in V_{h, p}}\left\|\boldsymbol{E}-\boldsymbol{v}_{h}\right\|_{\mathrm{DG}}+h^{\min \{t, p+1\}}\|\nabla \times \boldsymbol{E}\|_{H^{t}(\Omega)^{3}}\right) \\
& \leq C K\left(\left\|\boldsymbol{E}-\Pi_{N} \boldsymbol{E}\right\|_{\mathrm{DG}}+h^{\min \{t, p+1\}}\|\nabla \times \boldsymbol{E}\|_{H^{t}(\Omega)^{3}}\right) \\
& \leq C K h^{\min \{t, p\}}\left(\|\boldsymbol{E}\|_{H^{t}(\Omega)^{3}}+\|\nabla \times \boldsymbol{E}\|_{H^{t}(\Omega)^{3}}+\left\|\boldsymbol{E}_{T}\right\|_{H_{\|}^{t}(\Sigma)^{3}}+\left\|\nabla_{\Sigma} \times\left(\boldsymbol{E}_{T}\right)\right\|_{L^{2}(\Sigma)}\right),
\end{aligned}
$$

which proves the theorem. 
Remark 3.17. For large values of $\omega$ and for $\tau \approx \tau^{*}$, the ratio between continuity and coercivity constant and, hence, the constant $K$ in (3.24) behaves like

$$
\frac{\gamma_{\mathrm{DG}}(\omega)}{\alpha_{\mathrm{DG}}(\omega)} \sim \omega^{3}, \quad \omega \rightarrow \infty,
$$

which might seem to be suboptimal. This is due to the fact that we use the above defined standard graph norms $\|\cdot\|_{X}$ and $\|\cdot\|_{\text {DG }}$ on $X$ and $\widetilde{V}_{h, p}$, respectively, which are not scaled by $\omega$ (as often done in the literature). The reason for this choice is the fact that we use $\omega$ as a parameter within the RBM.

\section{REDUCED BASIS METHOD}

As already indicated above, we ultimately aim at constructing a numerical method that is able to rapidly compute a certified approximation to the electric field density $\boldsymbol{E}(\omega)$ as a function of the frequency $\omega$, in particular for many different values of $\omega$ - a so-called multi-query problem.

The main challenge here is that the mathematical properties of the underlying pde crucially depend on the value of $\omega$, not only - but also - all involved stability constants are heavily $\omega$-dependent. Typically, a Reduced Basis Method (RBM) requires a smooth (or at least a known) dependence of the solution $\boldsymbol{E}(\omega)$ w.r.t. the parameter $\omega$. This is not the case here. We will show that the above introduced hp-dG method is in fact a possible choice, which allows for a - more or less - standard RBM, which is shown in Section 5 below to be efficient and robust.

In the context of the RBM, the detailed, i.e., high dimensional approximation $\boldsymbol{E}_{h}(\omega)$ of the exact solution $\boldsymbol{E}(\omega)$ of $(2.5)$ is called truth approximation. The key to set up the formulation for a lower dimensional reduced basis approximation is the idea that the set of all possible truth approximations $\mathcal{M}^{\mathcal{N}}:=$ $\left\{\boldsymbol{E}_{h}(\omega)\right.$ solves $\left.(3.9) \mid \omega \in \mathcal{D}\right\}$ lies on a low-dimensional manifold in $V_{h, p}$, [30]. Instead of computing the -expensive- truth approximations $\boldsymbol{E}_{h}(\omega)$ for all frequencies $\omega$ in a given parameter domain $\mathcal{D}$, the RBM amounts to finding a suitable approximation space $X_{N} \subset V_{h, p}$ of $\mathcal{M}^{\mathcal{N}}$ with lower dimension $N:=\operatorname{dim}\left(X_{N}\right) \ll$ $\operatorname{dim}\left(V_{h, p}\right)=: \mathcal{N}=\mathcal{N}_{h, p}$ and then computing -cheap-approximations $\boldsymbol{E}_{N}(\omega) \in X_{N}$. This is done using snapshots, i.e., truth approximations for $N$ different values of the parameter, cf. [30],

$$
X_{N}:=\operatorname{span}\left\{\boldsymbol{E}_{h}(\omega) \text { solves }(3.9) \mid \omega \in S_{N}\right\}, \quad S_{N}:=\left\{\omega_{1}, \ldots, \omega_{N}\right\} \subset \mathcal{D} .
$$

In the online phase, one computes the Galerkin approximation in the previously determined low-dimensional space $X_{N}$, i.e. ${ }^{4}$

$$
a_{h}\left(\boldsymbol{E}_{N}(\omega), \boldsymbol{v}_{N} ; \omega\right)=f\left(\boldsymbol{v}_{N} ; \omega\right) \quad \forall \boldsymbol{v}_{N} \in X_{N} .
$$

The spaces $X_{N}$ are called reduced basis spaces, a standard procedure to construct them is a greedy algorithm, see $e . g$. $[7,30]$. This algorithm constructs the space $X_{N}$ iteratively by enriching it with one new basis function in each iteration. The particular choice of the basis functions is based upon an error indicator or estimator, the algorithm therefore depends on efficiently computable a posteriori error bounds $\left\|\boldsymbol{E}_{h}(\omega)-\boldsymbol{E}_{N}(\omega)\right\|_{\mathrm{DG}} \leq \Delta_{N}(\omega)$. The greedy procedure then maximizes the efficiently computable $\Delta_{N}(\omega)$ w.r.t. an appropriate training set $\Xi_{\text {train }} \subset \mathcal{D}$ to define the snapshots.

The key to these efficiently evaluable error bounds as well as to the efficiency of the calculation of the RBM solution $\boldsymbol{E}_{N}(\omega)$ is an affine dependency of $a_{h}$ and $f$ w.r.t. the parameter $\omega$, i.e., they must be of the following form:

$$
a_{h}\left(\boldsymbol{u}_{h}, \boldsymbol{v}_{h} ; \omega\right)=\sum_{q=1}^{Q_{a}} \Theta_{q}^{a}(\omega) a_{h}^{q}\left(\boldsymbol{u}_{h}, \boldsymbol{v}_{h}\right), \quad f\left(\boldsymbol{v}_{h} ; \omega\right)=\sum_{q=1}^{Q_{f}} \Theta_{q}^{f}(\omega) f^{q}\left(\boldsymbol{v}_{h}\right)
$$

for all $\boldsymbol{u}_{h}, \boldsymbol{v}_{h} \in V_{h, p}$ and all $\omega \in \mathcal{D}$ with parameter-dependent functions $\Theta_{q}^{a}(\omega), \Theta_{q}^{f}(\omega): \mathcal{D} \rightarrow \mathbb{R}$, as well as sesquilinear forms $a_{h}^{q}(\cdot, \cdot)$ and antilinear forms $f_{q}(\cdot)$ which are independent of the parameter $\omega$.

\footnotetext{
${ }^{4}$ To shorten notation we omit $\tau$ since it is chosen constant in our numerical experiments.
} 
The online-efficiency is obtained via precomputing the values $a_{h}^{q}\left(\boldsymbol{\xi}_{i}, \boldsymbol{\xi}_{j}\right), i, j \in\{1, \ldots, N\}, q \in\left\{1, \ldots, Q_{a}\right\}$ and $f^{q}\left(\boldsymbol{\xi}_{i}\right), i \in\{1, \ldots, N\}, q \in\left\{1, \ldots, Q_{f}\right\}$ in a precedent, possibly time-consuming offline phase, where $\left\{\boldsymbol{\xi}_{1}, \ldots, \boldsymbol{\xi}_{N}\right\}$ denotes a basis of the RB space $X_{N}$. The mentioned error estimator $\Delta_{N}(\omega)$ can, e.g., be based upon the dual norm of the RB residual $r_{N}(\cdot ; \omega)=f(\cdot ; \omega)-a_{h}\left(\boldsymbol{E}_{N}, \cdot ; \omega\right): V_{h, p} \rightarrow \mathbb{C}$ and the coercivity constant $\alpha_{\mathrm{DG}}(\omega)$ of $a_{h}(\cdot, \cdot ; \omega)$. It reads, cf. [30]:

$$
\Delta_{N}(\omega)=\frac{\left\|r_{N}(\cdot ; \omega)\right\|_{\mathrm{DG}}}{\alpha_{\mathrm{DG}}(\omega)}=\frac{1}{\alpha_{\mathrm{DG}}(\omega)} \sup _{\boldsymbol{v}_{h} \in V_{h, p}} \frac{r_{N}\left(\boldsymbol{v}_{h} ; \omega\right)}{\left\|\boldsymbol{v}_{h}\right\|_{\mathrm{DG}}}
$$

For $\omega \in \mathcal{D}$, the dual norm of $r_{N}(\cdot ; \omega)$ can be evaluated via the dG-norm of its Riesz representative $\boldsymbol{v}_{r_{N}}(\omega) \in V_{h, p}$ which satisfies $\left\|\boldsymbol{v}_{r_{N}}(\omega)\right\|_{\mathrm{DG}}=\left\|r_{N}(\cdot ; \omega)\right\|_{\mathrm{DG}^{*}}$. Based upon the affine decomposition of $a_{h}$ and $f$, also the norm of the Riesz representative $\boldsymbol{v}_{r_{N}}(\omega)$ is offline-online-decomposable $(e . g .[11,19,30])$ and can therefore be evaluated efficiently in the online phase. The coercivity constant $\alpha_{\mathrm{DG}}(\omega)$ can e.g. be computed by an eigenvalue problem or it can be approximated via the Successive Constraint Method (SCM), [23].

The above considerations indicate, that we can use the above introduced hp-dG discretization as a truth approximation within the RBM. Since the frequency $\omega$ serves as the parameter, the challenge remains that $\boldsymbol{E}_{h}(\omega)$ strongly depends on $\omega$.

\section{Numerical Results}

In this section, we present results of some numerical experiments for the investigated problem being treated with the RBM. As already mentioned, one crucial ingredient of the RBM is the affine decomposition of $a_{h}$ and $f$ w.r.t. the parameter. For our dG formulation (3.9), this affine form is readily given by:

$$
\begin{array}{rlrl}
\Theta_{1}^{a}(\omega) & : \equiv 1, & a_{1}\left(\boldsymbol{u}_{h}, \boldsymbol{v}_{h}\right):=\left(\mu^{-1} \nabla_{h} \times \boldsymbol{u}_{h}, \nabla_{h} \times \boldsymbol{v}_{h}\right)_{\Omega}-\left\langle\llbracket \boldsymbol{u}_{h} \rrbracket,\left\{\left\{\mu^{-1} \nabla_{h} \times \boldsymbol{v}_{h}\right\}\right\}\right\rangle_{\mathcal{F}_{h}^{I \cup \Gamma}} \\
& -\left\langle\left\{\left\{\mu^{-1} \nabla_{h} \times \boldsymbol{u}_{h}\right\}, \llbracket \boldsymbol{v}_{h} \rrbracket\right\rangle_{\mathcal{F}_{h}^{I \cup \Gamma}}+\left\langle\tau \mathrm{h}^{-1} \llbracket \boldsymbol{u}_{h} \rrbracket, \llbracket \boldsymbol{v}_{h} \rrbracket\right\rangle_{\mathcal{F}_{h}^{I \cup \Gamma}},\right. \\
\Theta_{2}^{a}(\omega):=\omega^{2}, & a_{2}\left(\boldsymbol{u}_{h}, \boldsymbol{v}_{h}\right):=-\left(\varepsilon \boldsymbol{u}_{h}, \boldsymbol{v}_{h}\right)_{\Omega}, \\
\Theta_{3}^{a}(\omega):=\omega, & a_{3}\left(\boldsymbol{u}_{h}, \boldsymbol{v}_{h}\right):=-i\left(\sigma \boldsymbol{u}_{h}, \boldsymbol{v}_{h}\right)_{\Omega}-i \lambda\left(\varepsilon_{0} \mu_{0}^{-1}\right)^{1 / 2}\left\langle\left(\boldsymbol{u}_{h}\right)_{T},\left(\boldsymbol{v}_{h}\right)_{T}\right\rangle_{\Sigma}, \\
\Theta_{1}^{f}(\omega):=\omega, & f_{1}\left(\boldsymbol{v}_{h}\right):=i \sqrt{\varepsilon_{0}}\left(\boldsymbol{J}_{\boldsymbol{a}}, \boldsymbol{v}_{h}\right)_{\Omega}, \\
\Theta_{2}^{f}(\omega): \equiv 1, & f_{2}\left(\boldsymbol{v}_{h}\right):=\left\langle\mu_{0}^{-1} \boldsymbol{g},\left(\boldsymbol{v}_{h}\right)_{T}\right\rangle_{\Sigma},
\end{array}
$$

and, therefore, $Q_{a}=3, Q_{f}=2$. Note, that $\boldsymbol{J}_{\boldsymbol{a}}$ and $\boldsymbol{g}$ are chosen to be parameter-independent. We use boundary conditions as specified in (2.1b) and (2.1c). The models we use for our numerical tests were created using COMSOL Multiphysics 4.2a. Details of the implementation an all data are given in Appendix B. All RB calculations were implemented in RBmatlab, see http://www.morepas.org.

As geometry we use the unit cube $(0,1)^{3}$ from which we cut out two smaller blocks, each of side length $1 / 4$. One of the smaller blocks is placed parallel to the large block, one is rotated by 45 degrees about the $z$-axis, see Figure 2. The two interior blocks are supposed to be perfectly conducting (2.1b), whereas an impedance boundary condition (2.1c) is imposed on the exterior boundary.

We implemented two versions of the model: Model 1 has constant coefficients $\mu \equiv \mu_{0}=4 \pi \times 10^{-7}, \varepsilon \equiv \varepsilon_{0}=$ $8.854 \times 10^{-12}$, and $\sigma \equiv 0.01$. In Model 2 the coefficients are given by $\mu(\boldsymbol{x})=\mu_{0}\left(1+\left\|\boldsymbol{x}-(0.5,0.5,0.5)^{T}\right\|\right), \varepsilon(\boldsymbol{x})=$ $\varepsilon_{0}(1+\|\boldsymbol{x}\|)$, and $\sigma(\boldsymbol{x})=0.01\left(1+0.5 x_{1}^{2}\right)$. Referring to [22], we chose $\tau=1000 / \mu_{0}$ for both models. With these two models, we performed a greedy sampling with a parameter domain $\mathcal{D}=[1,50] \mathrm{GHz}$ which was discretized into 97 equidistant sampling points to obtain $\Xi_{\text {train }}$.

Note also that we are dealing with complex-valued degrees of freedom (DOFs), which has to be taken into account when choosing a solver. Since the MATLAB backslash-solver (at least for finer discretizations) was not capable of an efficient numerical solution, we used MUMPS [3,4], which allows us to use about 300000 DOFs on an iMac, $3.2 \mathrm{GHz}$ Intel Core i3 with 8GB RAM. 


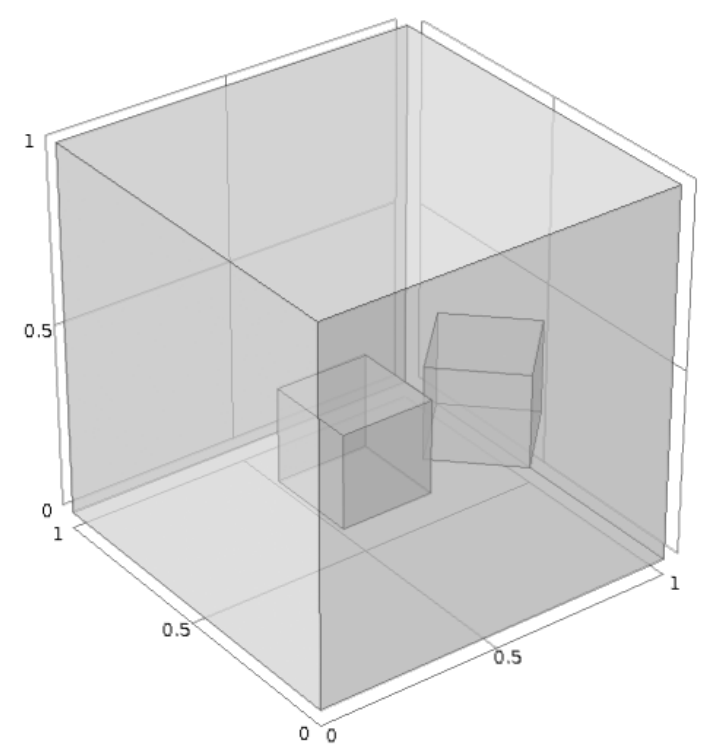

FiguRE 2. Geometry for the numerical experiments.

TABLE 1. $h$-convergence: $\left\|\boldsymbol{E}^{\star}-\boldsymbol{E}_{h}\right\|_{\mathrm{DG}}$ for different $h$.

\begin{tabular}{lrllll}
\hline \multicolumn{2}{c}{$p=1$} & \multicolumn{2}{c}{ Model 1 } & \multicolumn{2}{c}{ Model 2 } \\
\hline$h$ & \# DOFs & $\omega_{1}=1$ & $\omega_{2}=10$ & $\omega_{1}=1$ & $\omega_{2}=10$ \\
\hline 1 & 345 & 1.4637 & 2.5759 & 1.1366 & 4.3942 \\
$1 / 2$ & 2760 & 0.9465 & 0.6149 & 0.7506 & 1.1621 \\
$1 / 4$ & 22080 & 0.5653 & 0.2853 & 0.4521 & 0.7041 \\
$1 / 8$ & 176640 & 0.2886 & 0.1357 & 0.2286 & 0.2679 \\
\hline
\end{tabular}

In absence of an analytic solution to (2.1a)-(2.1c), we determine a reference solution $\boldsymbol{E}^{\star}$ and investigate $\left\|\boldsymbol{E}^{\star}-\boldsymbol{E}_{h}\right\|_{\text {DG }}$ for decreasing mesh size $h$ in order to validate our code w.r.t. (3.24). We start with $h=1$ and refine the mesh uniformly until we end up at a mesh size of $h=1 / 16$. Thus, we obtain conforming meshes. We choose $p=1$. The solution $\boldsymbol{E}_{1 / 16}$ consisted of 1413120 DOFs and was used as reference solution $\boldsymbol{E}^{\star}$, whose computation took about $7 \mathrm{~h}$. The $h$-convergence results are shown in Table 1.

For the greedy algorithm we used different mesh sizes that are pre-defined by COMSOL. The number of DOFs varies from 7818 to 290673 . In Figure 3 we show the convergence of the error during the greedy algorithm when using the real error (strong greedy)

$$
\max _{\omega \in \Xi_{\text {train }}}\left\|\boldsymbol{e}_{N}(\omega)\right\|_{\mathrm{DG}}, \quad \boldsymbol{e}_{N}(\omega):=\boldsymbol{E}_{h}(\omega)-\boldsymbol{E}_{N}(\omega)
$$

as well as the error estimator $\max _{\omega \in \Xi_{\text {train }}} \Delta_{N}(\omega)$ as error indicator. There are several versions of the Successive Constraint Method (SCM) for complex-valued problems, e.g. [11,19,20]. As proposed in [27], we instead obtained the coercivity constant via an interpolation method based on precalculated values of $\alpha_{\mathrm{DG}}(\omega)$.

We observe an exponential decay of the error. As expected, the decay is faster for Model 1 than for Model 2 which is the more sophisticated one. In addition, although the error estimator overestimates the real error, it reflects the dependency on the frequency $\omega$ correctly, cf. Figure 4, which is crucial for the selection of the snapshots during the greedy algorithm. For Model 1, the RBM matrices are almost singular and, hence, the reduced linear systems became unstable when creating reduced bases with more than 50 basis functions without 


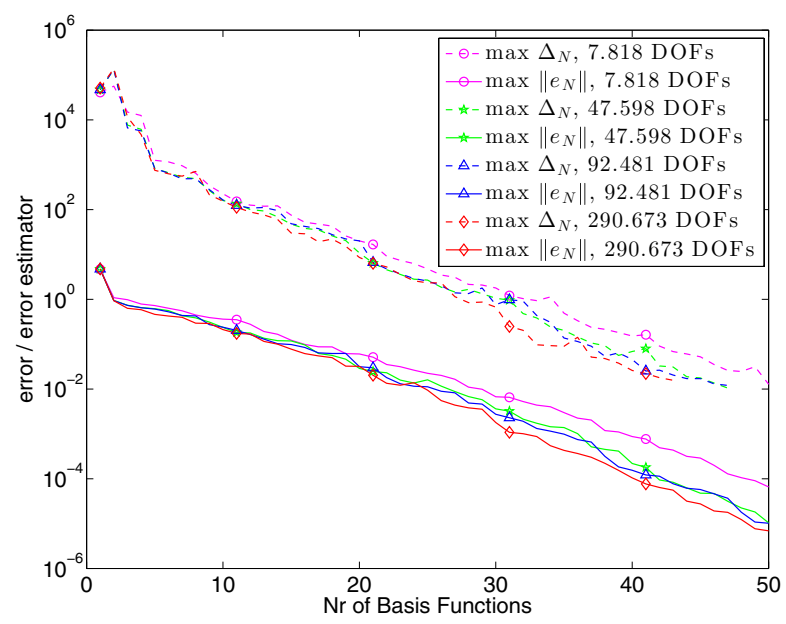

(a) Model 1

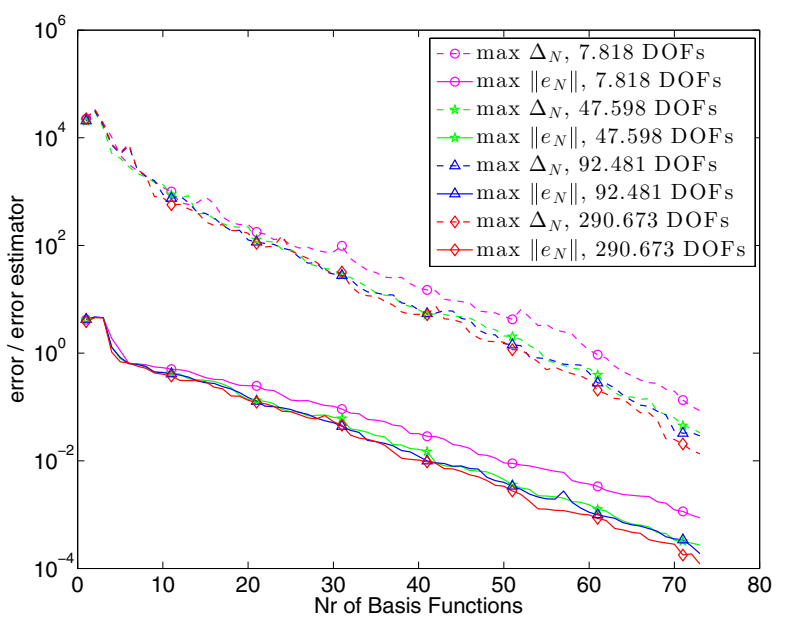

(b) Model 2

FIGURE 3. Real error and error estimator during the greedy algorithm, $p=1$.

TABLE 2. Runtimes.

\begin{tabular}{lcccccccc}
\hline \multicolumn{3}{c}{ Model 1 $(N=50)$} & \multicolumn{5}{c}{ Model 2 $(N=73)$} \\
\hline \# DOFs & 290673 & 92481 & 47598 & 7818 & 290673 & 92481 & 47598 & 7818 \\
$t_{\text {det. }}[\mathrm{s}]$ & 158.767 & 14.872 & 4.923 & 0.258 & 157.087 & 14.710 & 4.831 & 0.270 \\
$t_{\text {red. }}[\mathrm{s}]$ & 0.002 & 0.002 & 0.001 & 0.001 & 0.002 & 0.002 & 0.002 & 0.002 \\
speedup & 67449 & 9487 & 3648 & 219 & 75859 & 7280 & 2534 & 112 \\
\hline
\end{tabular}

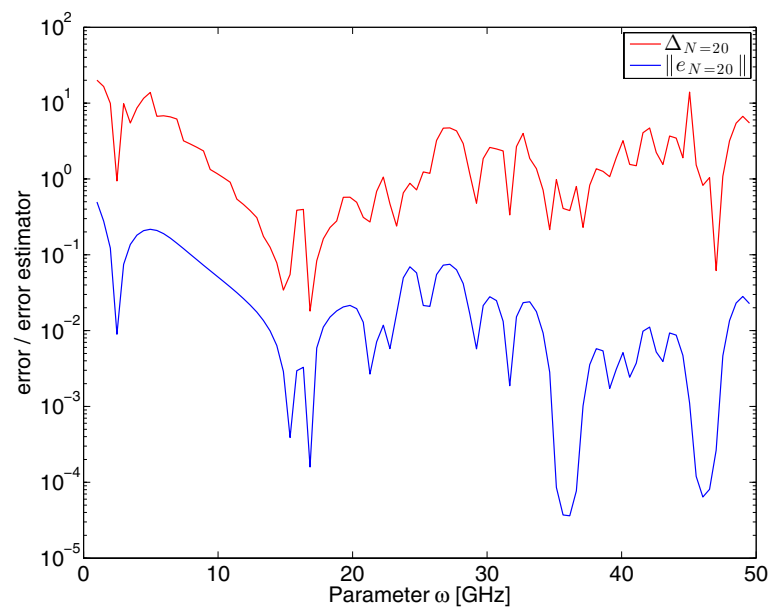

(a) Model 1, N=20.

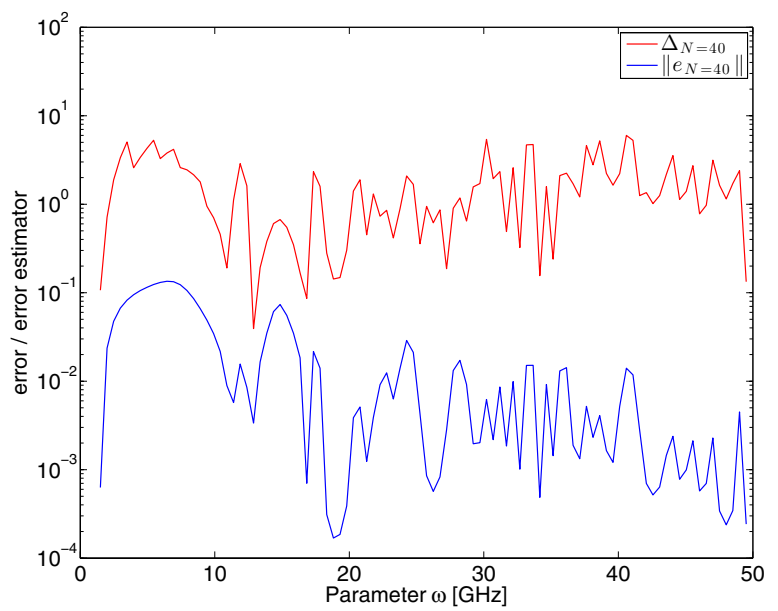

(b) Model 2, $\mathrm{N}=40$.

Figure 4. Error and error estimator for Model 1 with basis length $\mathrm{N}=20$ (left) and Model 2 with basis length $N=40$ (right). 
the computationally expensive Gram-Schmidt orthonormalisation. At this point, the error measured in the dG-norm $\|\cdot\|_{\text {DG }}$ was below $10^{-4}$. For Model 2, the bases became unstable for $N>73$ where the maximum error over the sampling set was at about $10^{-3}$. The offline phase took between $3 \mathrm{~min}$ for the coarse meshes and $18 \mathrm{~h}$ for the finer meshes. In Table 2, we show the online times needed for performing a detailed respectively reduced simulation. The online speedup factors vary from 112 to 75859 . One can also observe that for both models the error decays slightly faster for the finer discretized versions. This means that the physics of the problem can be represented better with a finer mesh and can therefore also be reproduced better with a reduced solution.

In order to verify the robustness of our RB approach, we finally investigate the dependency over the whole frequency range. In Figure 4, we show the error and error estimator over the whole parameter domain $\mathcal{D}$ with 100 equidistant grid points for the two models with 92481 DOFs and reduced bases with dimension $N=20$ (Model 1) resp. $N=40$ (Model 2). As desired, the error estimator resembles the behavior of the true error and both stay in an acceptable range, which shows the robustness of our dG discretization as well as of the RBM.

\section{Appendix A. Proofs}

\section{A.1. Proof of Theorem 2.3}

We collect detailed proofs of certain relevant results in this appendix.

Proof of Theorem 2.3. Fix $\omega>0$ and let $\boldsymbol{u}, \boldsymbol{v} \in X$, then

$$
\begin{aligned}
\left|a_{e}(\boldsymbol{u}, \boldsymbol{v} ; \omega)\right|= & \left|\left(\mu^{-1} \nabla \times \boldsymbol{u}, \nabla \times \boldsymbol{v}\right)_{\Omega}-\omega^{2}(\varepsilon \boldsymbol{u}, \boldsymbol{v})_{\Omega}-i \omega(\sigma \boldsymbol{u}, \boldsymbol{v})_{\Omega}-i \omega \lambda\left(\varepsilon_{0} \mu_{0}^{-1}\right)^{1 / 2}\left\langle\boldsymbol{u}_{T}, \boldsymbol{v}_{T}\right\rangle_{\Sigma}\right| \\
\leq & \mu_{-}^{-1}\|\nabla \times \boldsymbol{u}\|_{L^{2}(\Omega)^{3}}\|\nabla \times \boldsymbol{v}\|_{L^{2}(\Omega)^{3}}+\left(\omega^{2} \varepsilon_{+}+\omega \sigma_{+}\right)\|\boldsymbol{u}\|_{L^{2}(\Omega)^{3}}\|\boldsymbol{v}\|_{L^{2}(\Omega)^{3}} \\
& +\omega \lambda\left(\varepsilon_{0} \mu_{0}^{-1}\right)^{1 / 2}\left\|\boldsymbol{u}_{T}\right\|_{L^{2}(\Sigma)^{3}}\left\|\boldsymbol{v}_{T}\right\|_{L^{2}(\Sigma)^{3}} \\
\leq & \max \left\{\mu_{-}^{-1}, \omega^{2} \varepsilon_{+}+\omega \sigma_{+}, \omega \lambda\left(\varepsilon_{0} \mu_{0}^{-1}\right)^{1 / 2}\right\}\|\boldsymbol{u}\|_{X}\|\boldsymbol{v}\|_{X}=\gamma(\omega)\|\boldsymbol{u}\|_{X}\|\boldsymbol{v}\|_{X},
\end{aligned}
$$

which shows continuity. As for coercivity, we have:

$$
\begin{aligned}
\left|a_{e}(\boldsymbol{u}, \boldsymbol{u} ; \omega)\right|= & {\left[\left(\mu^{-1} \nabla \times \boldsymbol{u}, \nabla \times \boldsymbol{u}\right)_{\Omega}-\omega^{2}(\varepsilon \boldsymbol{u}, \boldsymbol{u})_{\Omega}\right)^{2} } \\
& \left.+\left(\omega(\sigma \boldsymbol{u}, \boldsymbol{u})_{\Omega}+\omega \lambda\left(\varepsilon_{0} \mu_{0}^{-1}\right)^{1 / 2}\left\|\boldsymbol{u}_{T}\right\|_{L^{2}(\Sigma)^{3}}^{2}\right)^{2}\right]^{1 / 2} \\
\geq & {\left[\left(\mu^{-1} \nabla \times \boldsymbol{u}, \nabla \times \boldsymbol{u}\right)_{\Omega}^{2}-2 \omega^{2}\left(\mu^{-1} \nabla \times \boldsymbol{u}, \nabla \times \boldsymbol{u}\right)_{\Omega}(\varepsilon \boldsymbol{u}, \boldsymbol{u})_{\Omega}\right.} \\
& \left.+\omega^{4}(\varepsilon \boldsymbol{u}, \boldsymbol{u})_{\Omega}^{2}+\omega^{2}(\sigma \boldsymbol{u}, \boldsymbol{u})_{\Omega}^{2}+\omega^{2} \lambda^{2} \varepsilon_{0} \mu_{0}^{-1}\left\|\boldsymbol{u}_{T}\right\|_{L^{2}(\Sigma)^{3}}^{4}\right]^{1 / 2} \\
\geq & {\left[(1-\delta)\left(\mu^{-1} \nabla \times \boldsymbol{u}, \nabla \times \boldsymbol{u}\right)_{\Omega}^{2}+\left(1-\delta^{-1}\right) \omega^{4}(\varepsilon \boldsymbol{u}, \boldsymbol{u})_{\Omega}^{2}\right.} \\
& \left.+\omega^{2}(\sigma \boldsymbol{u}, \boldsymbol{u})_{\Omega}^{2}+\omega^{2} \lambda^{2} \varepsilon_{0} \mu_{0}^{-1}\left\|\boldsymbol{u}_{T}\right\|_{L^{2}(\Sigma)^{3}}^{4}\right]^{1 / 2},
\end{aligned}
$$

for $\delta \in(0,1)$ by Young's inequality. The fact that $\sqrt{a+b} \geq \frac{1}{\sqrt{2}}(\sqrt{a}+\sqrt{b})$ for $a, b \geq 0$, together with $(2.2),(2.3)$ and (2.4) in Assumption 2.1, leads to

$$
\begin{aligned}
\left|a_{e}(\boldsymbol{u}, \boldsymbol{u} ; \omega)\right| \geq & \frac{1}{\sqrt{2}}\left[(1-\delta)\left(\mu^{-1} \nabla \times \boldsymbol{u}, \nabla \times \boldsymbol{u}\right)_{\Omega}^{2}+\left(1-\delta^{-1}\right) \omega^{4}(\varepsilon \boldsymbol{u}, \boldsymbol{u})_{\Omega}^{2}+\omega^{2}(\sigma \boldsymbol{u}, \boldsymbol{u})_{\Omega}^{2}\right]^{1 / 2} \\
& +\frac{\omega \lambda \sqrt{\varepsilon_{0}}}{\sqrt{2 \mu_{0}}}\left\|\boldsymbol{u}_{T}\right\|_{L^{2}(\Sigma)^{3}}^{2} \\
\geq & \frac{1}{\sqrt{2}}\left[\frac{1-\delta}{\mu_{+}^{2}}\|\nabla \times \boldsymbol{u}\|_{L^{2}(\Omega)^{3}}^{4}+\left(\omega^{2} \sigma_{-}^{2}-\left(\delta^{-1}-1\right) \omega^{4} \varepsilon_{+}^{2}\right)\|\boldsymbol{u}\|_{L^{2}(\Omega)^{3}}^{4}\right]^{\frac{1}{2}}+\frac{\omega \lambda \sqrt{\varepsilon_{0}}}{\sqrt{2 \mu_{0}}}\left\|\boldsymbol{u}_{T}\right\|_{L^{2}(\Sigma)^{3}}^{2} .
\end{aligned}
$$


Choose $1>\delta>\frac{\omega^{2} \varepsilon_{+}^{2}}{\omega^{2} \varepsilon_{+}^{2}+\sigma_{-}^{2}}>0$, e.g., $\delta=\frac{\omega^{2} \varepsilon_{+}^{2}+\sigma_{-}^{2} / 2}{\omega^{2} \varepsilon_{+}^{2}+\sigma_{-}^{2}}$,

$$
\begin{aligned}
\left|a_{e}(\boldsymbol{u}, \boldsymbol{u} ; \omega)\right| \geq & \frac{1}{\sqrt{2}}\left[\frac{\sigma_{-}^{2}}{2\left(\omega^{2} \varepsilon_{+}^{2}+\sigma_{-}^{2}\right) \mu_{+}^{2}}\|\nabla \times \boldsymbol{u}\|_{L^{2}(\Omega)^{3}}^{4}+\frac{\omega^{4} \varepsilon_{+}^{2} \sigma_{-}^{2}+\omega^{2} \sigma_{-}^{4}}{2 \omega^{2} \varepsilon_{+}^{2}+\sigma_{-}^{2}}\|\boldsymbol{u}\|_{L^{2}(\Omega)^{3}}^{4}\right]^{\frac{1}{2}}+\frac{\omega \lambda \sqrt{\varepsilon_{0}}}{\sqrt{2 \mu_{0}}}\left\|\boldsymbol{u}_{T}\right\|_{L^{2}(\Sigma)^{3}}^{2} \\
\geq & \frac{1}{2}\left(\frac{\sigma_{-}^{2}}{2\left(\omega^{2} \varepsilon_{+}^{2}+\sigma_{-}^{2}\right) \mu_{+}^{2}}\right)^{\frac{1}{2}}\|\nabla \times \boldsymbol{u}\|_{L^{2}(\Omega)^{3}}^{2} \\
& +\frac{\omega}{2}\left(\frac{\omega^{2} \varepsilon_{+}^{2} \sigma_{-}^{2}+\sigma_{-}^{4}}{2 \omega^{2} \varepsilon_{+}^{2}+\sigma_{-}^{2}}\right)^{\frac{1}{2}}\|\boldsymbol{u}\|_{L^{2}(\Omega)^{3}}^{2}+\frac{\omega \lambda \sqrt{\varepsilon_{0}}}{\sqrt{2 \mu_{0}}}\left\|\boldsymbol{u}_{T}\right\|_{L^{2}(\Sigma)^{3}}^{2} \\
\geq & \min \left\{\frac{\sigma_{-}}{2^{3 / 2} \mu_{+}\left(\omega^{2} \varepsilon_{+}^{2}+\sigma_{-}^{2}\right)^{1 / 2}}, \frac{\omega}{2}\left(\frac{\omega^{2} \varepsilon_{+}^{2} \sigma_{-}^{2}+\sigma_{-}^{4}}{2 \omega^{2} \varepsilon_{+}^{2}+\sigma_{-}^{2}}\right)^{\frac{1}{2}}, \frac{\omega \lambda \sqrt{\varepsilon_{0}}}{\sqrt{2 \mu_{0}}}\right\}\|\boldsymbol{u}\|_{X}^{2} \\
= & \alpha(\omega)\|\boldsymbol{u}\|_{X}^{2} .
\end{aligned}
$$

For the right-hand side, standard arguments yield $|f(\boldsymbol{v} ; \omega)| \leq\left(\omega \sqrt{\varepsilon_{0}}\left\|\boldsymbol{J}_{\boldsymbol{a}}\right\|_{L^{2}(\Omega)^{3}}+\mu_{0}^{-1}\|\boldsymbol{g}\|_{L^{2}(\Sigma)^{3}}\right)\|\boldsymbol{v}\|_{X}=$ $C_{f}(\omega)\|\boldsymbol{v}\|_{X}$ for all $\boldsymbol{v} \in X$, which proves the claim.

\section{A.2. Proof of Theorem 3.2}

Proof of Theorem 3.2. The proof follows the standard method to show consistency of discrete dG variational formulations that is mentioned, e.g., in [31] for other fluxes. Since, however, we were not able to find a proof in the literature for the fluxes specified in Section 3.1, we state it here completely.

If $\boldsymbol{E}$ is a solution of (2.1a)-(2.1c), then the following tangential jumps vanish, $\llbracket \mu^{-1} \nabla \times \boldsymbol{E} \rrbracket=0$ on $\mathcal{F}_{h}^{I}$, $\llbracket \boldsymbol{E} \rrbracket=0$ on $\mathcal{F}_{h}^{I} \cup \mathcal{F}_{h}^{\Gamma}$, since $\boldsymbol{E}$ and $\mu^{-1} \nabla \times \boldsymbol{E}$ are functions in $H$ (curl, $\left.\Omega\right)$ and $\boldsymbol{n} \times \boldsymbol{E}=0$ on $\Gamma_{1}, \ldots, \Gamma_{M-1}$. This fact together with the identity $\left(\mu^{-1} \nabla \times \boldsymbol{E}, \nabla_{h} \times \boldsymbol{v}_{h}\right)_{\Omega}=\left(\nabla \times\left(\mu^{-1} \nabla \times \boldsymbol{E}\right), \boldsymbol{v}_{h}\right)_{\Omega}-\left\langle\llbracket \mu^{-1} \nabla \times \boldsymbol{E} \rrbracket,\left\{\left\{\boldsymbol{v}_{h}\right\}\right\}\right\rangle_{\mathcal{F}_{h}^{I}}+$ $\left\langle\left\{\left\{\mu^{-1} \nabla \times \boldsymbol{E}\right\}\right\}, \llbracket \boldsymbol{v}_{h} \rrbracket\right\rangle_{\mathcal{F}_{h}^{I}}-\left\langle\boldsymbol{n} \times\left(\mu^{-1} \nabla \times \boldsymbol{E}\right), \boldsymbol{v}_{h}\right\rangle_{\mathcal{F}_{h}^{B}}$ yield the desired consistency:

$$
\begin{aligned}
a_{h}\left(\boldsymbol{E}, \boldsymbol{v}_{h} ; \omega, \tau\right)= & \left(\nabla \times\left(\mu^{-1} \nabla \times \boldsymbol{E}\right), \boldsymbol{v}_{h}\right)_{\Omega}-\overbrace{\left\langle\llbracket \mu^{-1} \nabla \times \boldsymbol{E} \rrbracket,\left\{\left\{\boldsymbol{v}_{h}\right\}\right\}\right\rangle_{\mathcal{F}_{h}^{I}}}^{=0} \\
& +\left\langle\left\{\left\{\mu^{-1} \nabla \times \boldsymbol{E}\right\}\right\}, \llbracket \boldsymbol{v}_{h} \rrbracket\right\rangle_{\mathcal{F}_{h}^{I}}-\left\langle\boldsymbol{n} \times\left(\mu^{-1} \nabla \times \boldsymbol{E}\right), \boldsymbol{v}_{h}\right\rangle_{\mathcal{F}_{h}^{I}}-\left\langle\boldsymbol{n} \times\left(\mu^{-1} \nabla \times \boldsymbol{E}\right), \boldsymbol{v}_{h}\right\rangle_{\mathcal{F}_{h}} \\
& -\left(\left(\omega^{2} \varepsilon+i \omega \sigma\right) \boldsymbol{E}, \boldsymbol{v}_{h}\right)_{\Omega}-i \omega \lambda\left(\varepsilon_{0} \mu_{0}^{-1}\right)^{1 / 2}\left\langle\boldsymbol{E}_{T},\left(\boldsymbol{v}_{h}\right)_{T}\right\rangle_{\Sigma}-\left\langle\left\{\left\{\mu^{-1} \nabla \times \boldsymbol{E}\right\}, \llbracket \boldsymbol{v}_{h} \rrbracket\right\rangle_{\mathcal{F}_{h}^{I \cup \Gamma}}\right. \\
& -\underbrace{\left\langle\llbracket \boldsymbol{E} \rrbracket,\left\{\left\{\mu^{-1} \nabla_{h} \times \boldsymbol{v}_{h}\right\}\right\}\right\rangle_{\mathcal{F}_{h}^{I \cup \Gamma}}}_{=0}+\underbrace{\left\langle\tau h^{-1} \llbracket \boldsymbol{E} \rrbracket, \llbracket \boldsymbol{v}_{h} \rrbracket\right\rangle_{\mathcal{F}_{h}^{I \cup T}}}_{=0} \\
= & \left(\nabla \times\left(\mu^{-1} \nabla \times \boldsymbol{E}\right)-\left(\omega^{2} \varepsilon+i \omega \sigma\right) \boldsymbol{E}, \boldsymbol{v}_{h}\right)_{\Omega}-\left\langle\boldsymbol{n} \times\left(\mu^{-1} \nabla \times \boldsymbol{E}\right),\left(\boldsymbol{v}_{h}\right)_{T}\right\rangle_{\Sigma} \\
& -i \omega \lambda\left(\varepsilon_{0} \mu_{0}^{-1}\right)^{1 / 2}\left\langle\boldsymbol{E}_{T},\left(\boldsymbol{v}_{h}\right)_{T}\right\rangle_{\Sigma} \\
= & i \omega \sqrt{\varepsilon_{0}}\left(\boldsymbol{J}_{\boldsymbol{a}}, \boldsymbol{v}_{h}\right)_{\Omega}+\mu_{0}^{-1}\left\langle\boldsymbol{g},\left(\boldsymbol{v}_{h}\right)_{T}\right\rangle_{\Sigma}=f\left(\boldsymbol{v}_{h} ; \omega\right)
\end{aligned}
$$

for all $\boldsymbol{v}_{h} \in V_{h, p}$, since $\left\langle\boldsymbol{n} \times\left(\mu^{-1} \nabla \times \boldsymbol{E}\right), \boldsymbol{v}_{h}\right\rangle_{\mathcal{F}_{h}}=\left\langle\boldsymbol{n} \times\left(\mu^{-1} \nabla \times \boldsymbol{E}\right),\left(\boldsymbol{v}_{h}\right)_{T}\right\rangle_{\Sigma}$. 


\section{A.3. Proof of Theorem 3.7}

Proof of Theorem 3.7. Fix $\omega, \tau>0$ and let $\boldsymbol{u}, \boldsymbol{v} \in \widetilde{V}_{h, p}$. The properties (2.2), (2.3), (2.4) of $\mu, \varepsilon$ and $\sigma$ yield

$$
\begin{aligned}
& \left|\widetilde{a}_{h}(\boldsymbol{u}, \boldsymbol{v} ; \omega, \tau)\right|=\mid\left(\mu^{-1} \nabla_{h} \times \boldsymbol{u}, \nabla_{h} \times \boldsymbol{v}\right)_{\Omega}-\omega^{2}(\varepsilon \boldsymbol{u}, \boldsymbol{v})_{\Omega}-i \omega(\sigma \boldsymbol{u}, \boldsymbol{v})_{\Omega} \\
& -\left(\mathcal{L}_{\mu}(\boldsymbol{u}), \mu^{-1} \nabla_{h} \times \boldsymbol{v}\right)_{\Omega}-\left(\mu^{-1} \nabla_{h} \times \boldsymbol{u}, \mathcal{L}_{\mu}(\boldsymbol{v})\right)_{\Omega} \\
& +\left\langle\tau \mathrm{h}^{-1} \llbracket \boldsymbol{u} \rrbracket, \llbracket \boldsymbol{v} \rrbracket\right\rangle_{\mathcal{F}_{h}^{I \cup \Gamma}}-i \omega \lambda\left(\varepsilon_{0} \mu_{0}^{-1}\right)^{1 / 2}\left\langle\boldsymbol{u}_{T}, \boldsymbol{v}_{T}\right\rangle_{\Sigma} \mid \\
& \leq \mu_{-}^{-1}\left\|\nabla_{h} \times \boldsymbol{u}\right\|_{L^{2}(\Omega)^{3}}\left\|\nabla_{h} \times \boldsymbol{v}\right\|_{L^{2}(\Omega)^{3}}+\left(\omega^{2} \varepsilon_{+}+\omega \sigma_{+}\right)\|\boldsymbol{u}\|_{L^{2}(\Omega)^{3}}\|\boldsymbol{v}\|_{L^{2}(\Omega)^{3}} \\
& +\left\|\mu^{-1} \mathcal{L}_{\mu}(\boldsymbol{u})\right\|_{L^{2}(\Omega)^{3}}\left\|\nabla_{h} \times \boldsymbol{v}\right\|_{L^{2}(\Omega)^{3}}+\left\|\nabla_{h} \times \boldsymbol{u}\right\|_{L^{2}(\Omega)^{3}}\left\|\mu^{-1} \mathcal{L}_{\mu}(\boldsymbol{v})\right\|_{L^{2}(\Omega)^{3}} \\
& +\tau\left\|\mathrm{h}^{-\frac{1}{2}} \llbracket \boldsymbol{u} \rrbracket\right\|_{\mathcal{F}_{h}^{I \cup \Gamma}}\left\|\mathrm{h}^{-\frac{1}{2}} \llbracket \boldsymbol{v} \rrbracket\right\|_{\mathcal{F}_{h}^{I \cup \Gamma}}+\omega \lambda\left(\varepsilon_{0} \mu_{0}^{-1}\right)^{1 / 2}\left\|\boldsymbol{u}_{T}\right\|_{L^{2}(\Sigma)^{3}}\left\|\boldsymbol{v}_{T}\right\|_{L^{2}(\Sigma)^{3}} \\
& \leq \max \left\{\mu_{-}^{-1}+\frac{\mu_{+} \sqrt{C_{\mathrm{inv}}}}{\mu_{-}^{2}}, \omega^{2} \varepsilon_{+}+\omega \sigma_{+}, \tau+\frac{\mu_{+} \sqrt{C_{\mathrm{inv}}}}{\mu_{-}^{2}}, \omega \lambda\left[\frac{\varepsilon_{0}}{\mu_{0}}\right]^{\frac{1}{2}}\right\}\|\boldsymbol{u}\|_{\mathrm{DG}}\|\boldsymbol{v}\|_{\mathrm{DG}} \\
& =\gamma_{\mathrm{DG}}(\omega)\|\boldsymbol{u}\|_{\mathrm{DG}}\|\boldsymbol{v}\|_{\mathrm{DG}},
\end{aligned}
$$

because we can estimate as follows ( $\operatorname{since} \mathcal{L}_{\mu}(\boldsymbol{u}) \in V_{h, p}$ and $\Pi_{\mu} \boldsymbol{w} \in V_{h, p}$ )

$$
\begin{aligned}
\left\|\mu^{-1} \mathcal{L}_{\mu}(\boldsymbol{u})\right\|_{L^{2}(\Omega)^{3}} & =\sup _{\boldsymbol{w} \in L^{2}(\Omega)^{3}} \frac{\left(\mu^{-1} \mathcal{L}_{\mu}(\boldsymbol{u}), \boldsymbol{w}\right)_{\Omega}}{\|\boldsymbol{w}\|_{L^{2}(\Omega)^{3}}}=\sup _{\boldsymbol{w} \in L^{2}(\Omega)^{3}} \frac{\left(\mathcal{L}_{\mu}(\boldsymbol{u}), \mu^{-1} \Pi_{\mu} \boldsymbol{w}\right)_{\Omega}}{\|\boldsymbol{w}\|_{L^{2}(\Omega)^{3}}} \\
& =\sup _{\boldsymbol{w} \in L^{2}(\Omega)^{3}} \frac{\left\langle\llbracket \boldsymbol{u} \rrbracket,\left\{\left\{\mu^{-1} \Pi_{\mu} \boldsymbol{w}\right\}\right\}\right\rangle_{\mathcal{F}_{h}^{I \cup \Gamma}}^{I \cup}}{\|\boldsymbol{w}\|_{L^{2}(\Omega)^{3}}} \leq\left\|\mathrm{h}^{-\frac{1}{2}} \llbracket \boldsymbol{u} \rrbracket\right\|_{\mathcal{F}_{h}^{I \cup \Gamma}} \sup _{\boldsymbol{w} \in L^{2}(\Omega)^{3}} \frac{\left\|\mathrm{h}^{\frac{1}{2}} \mu^{-1}\left\{\left\{\Pi_{\mu} \boldsymbol{w}\right\}\right\}\right\|_{\mathcal{F}_{h}^{I \cup \Gamma}}}{\|\boldsymbol{w}\|_{L^{2}(\Omega)^{3}}} \\
& \leq \mu_{-}^{-1} \sqrt{C_{\mathrm{inv}}}\left\|\mathrm{h}^{-\frac{1}{2}} \llbracket \boldsymbol{u}\right\|_{\mathcal{F}_{h}^{I \cup \Gamma}} \sup _{\boldsymbol{w} \in L^{2}(\Omega)^{3}} \frac{\left\|\Pi_{\mu} \boldsymbol{w}\right\|_{L^{2}(\Omega)^{3}}}{\|\boldsymbol{w}\|_{L^{2}(\Omega)^{3}}} \leq \mu_{+} \mu_{-}^{-2} \sqrt{C_{\mathrm{inv}}}\left\|\mathrm{h}^{-\frac{1}{2}} \llbracket \boldsymbol{u} \rrbracket\right\|_{\mathcal{F}_{h}^{I \cup \Gamma} .} .
\end{aligned}
$$

In this calculation we used the definitions (3.14) and (3.15) of the operators $\mathcal{L}_{\mu}$ and $\Pi_{\mu}$ as well as the estimates (3.13) and (3.16). This proves the assertion.

\section{A.4. Proof of Theorem 3.9}

Proof of Theorem 3.9. Fix $\omega>0$ and let $\boldsymbol{v}_{h} \in V_{h, p}$. Then,

$$
\begin{aligned}
\left|a_{h}\left(\boldsymbol{v}_{h}, \boldsymbol{v}_{h} ; \omega, \tau\right)\right|= & \mid\left(\mu^{-1} \nabla_{h} \times \boldsymbol{v}_{h}, \nabla_{h} \times \boldsymbol{v}_{h}\right)_{\Omega}-\omega^{2}\left(\varepsilon \boldsymbol{v}_{h}, \boldsymbol{v}_{h}\right)_{\Omega}-i \omega\left(\sigma \boldsymbol{v}_{h}, \boldsymbol{v}_{h}\right)_{\Omega} \\
& -\left\langle\llbracket \boldsymbol{v}_{h} \rrbracket,\left\{\left\{\mu^{-1} \nabla_{h} \times \boldsymbol{v}_{h}\right\}\right\}\right\rangle_{\mathcal{F}_{h}^{I \cup \Gamma}}-\left\langle\left\{\left\{\mu^{-1} \nabla_{h} \times \boldsymbol{v}_{h}\right\}, \llbracket \llbracket \boldsymbol{v}_{h} \rrbracket\right\rangle_{\mathcal{F}_{h}^{I \cup \Gamma}}\right. \\
& +\left\langle\tau \mathrm{h}^{-1} \llbracket \boldsymbol{v}_{h} \rrbracket, \llbracket \boldsymbol{v}_{h} \rrbracket\right\rangle_{\mathcal{F}_{h}^{I \cup \Gamma}}-i \omega \lambda\left(\varepsilon_{0} \mu_{0}^{-1}\right)^{1 / 2}\left\|\left(\boldsymbol{v}_{h}\right)_{T}\right\|_{L^{2}(\Sigma)^{3}}^{2} \mid .
\end{aligned}
$$

First, we use the inverse triangle inequality and Hölder's inequality to obtain

$$
\left|a_{h}\left(\boldsymbol{v}_{h}, \boldsymbol{v}_{h} ; \omega, \tau\right)\right| \geq \mid\left(\mu^{-1} \nabla_{h} \times \boldsymbol{v}_{h}, \nabla_{h} \times \boldsymbol{v}_{h}\right)_{\Omega}-\omega^{2}\left(\varepsilon \boldsymbol{v}_{h}, \boldsymbol{v}_{h}\right)_{\Omega}
$$




$$
\begin{aligned}
& +\tau\left\|\mathrm{h}^{-\frac{1}{2}} \llbracket \boldsymbol{v}_{h} \rrbracket\right\|_{\mathcal{F}_{h}^{I \cup \Gamma}}^{2}-i \omega\left(\sigma \boldsymbol{v}_{h}, \boldsymbol{v}_{h}\right)_{\Omega}-i \omega \lambda\left(\varepsilon_{0} \mu_{0}^{-1}\right)^{1 / 2}\left\|\left(\boldsymbol{v}_{h}\right)_{T}\right\|_{L^{2}(\Sigma)^{3}}^{2} \mid \\
& -2\left\|\mathrm{~h}^{-\frac{1}{2}} \llbracket \boldsymbol{v}_{h} \rrbracket\right\|_{\mathcal{F}_{h}^{I \cup \Gamma}}\left\|\mathrm{h}^{\frac{1}{2}}\left\{\left\{\mu^{-1} \nabla_{h} \times \boldsymbol{v}_{h}\right\}\right\}\right\|_{\mathcal{F}_{h}^{I \cup \Gamma}} .
\end{aligned}
$$

The definition of the absolute value of complex numbers yields

$$
\begin{aligned}
= & \left(\left[\left(\mu^{-1} \nabla_{h} \times \boldsymbol{v}_{h}, \nabla_{h} \times \boldsymbol{v}_{h}\right)_{\Omega}-\omega^{2}\left(\varepsilon \boldsymbol{v}_{h}, \boldsymbol{v}_{h}\right)_{\Omega}+\tau\left\|\mathrm{h}^{-\frac{1}{2}} \llbracket \boldsymbol{v}_{h} \rrbracket\right\|_{\mathcal{F}_{h}^{I \cup I}}^{2}\right]^{2}\right. \\
& +\left[\omega^{2}\left(\sigma \boldsymbol{v}_{h}, \boldsymbol{v}_{h}\right)_{\Omega}^{2}+2 \omega^{2} \lambda\left(\varepsilon_{0} \mu_{0}^{-1}\right)^{1 / 2}\left(\sigma \boldsymbol{v}_{h}, \boldsymbol{v}_{h}\right)_{\Omega}\left\|\left(\boldsymbol{v}_{h}\right)_{T}\right\|_{L^{2}(\Sigma)^{3}}^{2}\right. \\
& \left.\left.+\omega^{2} \lambda^{2} \varepsilon_{0} \mu_{0}^{-1}\left\|\left(\boldsymbol{v}_{h}\right)_{T}\right\|_{L^{2}(\Sigma)^{3}}^{4}\right]\right)^{\frac{1}{2}}-2\left\|\mathrm{~h}^{-\frac{1}{2}} \llbracket \boldsymbol{v}_{h} \rrbracket\right\|_{\mathcal{F}_{h}^{I \cup \Gamma}}\left\|\mathrm{h}^{\frac{1}{2}} \mu^{-1}\left\{\left\{\nabla_{h} \times \boldsymbol{v}_{h}\right\}\right\}\right\|_{\mathcal{F}_{h}^{I \cup \Gamma}} .
\end{aligned}
$$

Now we use property (2.2) of $\mu$

$$
\begin{aligned}
& \geq\left(\left[\left(\mu^{-1} \nabla_{h} \times \boldsymbol{v}_{h}, \nabla_{h} \times \boldsymbol{v}_{h}\right)_{\Omega}+\tau\left\|\mathrm{h}^{-\frac{1}{2}} \llbracket \boldsymbol{v}_{h} \rrbracket\right\|_{\mathcal{F}_{h}^{I \cup \Gamma}}^{2}\right]^{2}\right. \\
& -2 \omega^{2}\left(\varepsilon \boldsymbol{v}_{h}, \boldsymbol{v}_{h}\right)_{\Omega}\left[\left(\mu^{-1} \nabla_{h} \times \boldsymbol{v}_{h}, \nabla_{h} \times \boldsymbol{v}_{h}\right)_{\Omega}+\tau\left\|\mathrm{h}^{-\frac{1}{2}} \llbracket \boldsymbol{v}_{h} \rrbracket\right\|_{\mathcal{F}_{h}^{I \cup \Gamma}}^{2}\right] \\
& \left.+\omega^{4}\left(\varepsilon \boldsymbol{v}_{h}, \boldsymbol{v}_{h}\right)_{\Omega}^{2}+\omega^{2}\left(\sigma \boldsymbol{v}_{h}, \boldsymbol{v}_{h}\right)_{\Omega}^{2}+\omega^{2} \lambda^{2} \varepsilon_{0} \mu_{0}^{-1}\left\|\left(\boldsymbol{v}_{h}\right)_{T}\right\|_{L^{2}(\Sigma)^{3}}^{4}\right)^{1 / 2} \\
& -2 \mu_{-}^{-1}\left\|\mathrm{~h}^{-\frac{1}{2}} \llbracket \boldsymbol{v}_{h} \rrbracket\right\|_{\mathcal{F}_{h}^{I \cup \Gamma}}\left\|\mathrm{h}^{\frac{1}{2}}\left\{\left\{\nabla_{h} \times \boldsymbol{v}_{h}\right\}\right\}\right\|_{\mathcal{F}_{h}^{I \cup \Gamma}}
\end{aligned}
$$

as well as $\sqrt{x+y} \geq \frac{1}{\sqrt{2}}(\sqrt{x}+\sqrt{y})$ for $x, y \geq 0$ and we apply Young's inequality twice, for $a \in(0,1), \delta>0$

$$
\begin{aligned}
\geq & \frac{1}{\sqrt{2}}\left((1-a)\left[\left(\mu^{-1} \nabla_{h} \times \boldsymbol{v}_{h}, \nabla_{h} \times \boldsymbol{v}_{h}\right)_{\Omega}+\tau\left\|\mathrm{h}^{-\frac{1}{2}} \llbracket \boldsymbol{v}_{h} \rrbracket\right\|_{\mathcal{F}_{h}^{I \cup T}}^{2}\right]^{2}\right. \\
& \left.-\left(a^{-1}-1\right) \omega^{4}\left(\varepsilon \boldsymbol{v}_{h}, \boldsymbol{v}_{h}\right)_{\Omega}^{2}+\omega^{2}\left(\sigma \boldsymbol{v}_{h}, \boldsymbol{v}_{h}\right)_{\Omega}^{2}\right)^{1 / 2}+\frac{\omega \lambda \sqrt{\varepsilon_{0}}}{\sqrt{2 \mu_{0}}}\left\|\left(\boldsymbol{v}_{h}\right)_{T}\right\|_{L^{2}(\Sigma)^{3}}^{2} \\
& -\delta^{-1}\left\|\mathrm{~h}^{-\frac{1}{2}} \llbracket \boldsymbol{v}_{h} \rrbracket\right\|_{\mathcal{F}_{h}^{I \cup \Gamma}}^{2}-\delta \mu_{-}^{-2}\left\|\mathrm{~h}^{\frac{1}{2}}\left\{\left\{\nabla_{h} \times \boldsymbol{v}_{h}\right\}\right\}\right\|_{\mathcal{F}_{h}^{I \cup \Gamma}}^{2} .
\end{aligned}
$$

We note that $\left(a^{-1}-1\right)>0$ for $a \in(0,1)$ and use the properties (2.3) and (2.4) of $\varepsilon$ and $\sigma$ in Assumption 2.1 as well as the inverse inequality (3.13):

$$
\begin{aligned}
\geq & \frac{1}{\sqrt{2}}\left((1-a)\left[\left(\mu^{-1} \nabla_{h} \times \boldsymbol{v}_{h}, \nabla_{h} \times \boldsymbol{v}_{h}\right)_{\Omega}+\tau\left\|\mathrm{h}^{-\frac{1}{2}} \llbracket \boldsymbol{v}_{h} \rrbracket\right\|_{\mathcal{F}_{h}^{I \cup T}}^{2}\right]^{2}\right. \\
& \left.+\omega^{2}\left(\sigma_{-}^{2}-\left(a^{-1}-1\right) \omega^{2} \varepsilon_{+}^{2}\right)\left\|\boldsymbol{v}_{h}\right\|_{L^{2}(\Omega)^{3}}^{4}\right)^{1 / 2}+\frac{\omega \lambda \sqrt{\varepsilon_{0}}}{\sqrt{2 \mu_{0}}}\left\|\left(\boldsymbol{v}_{h}\right)_{T}\right\|_{L^{2}(\Sigma)^{3}}^{2} \\
& -\delta^{-1}\left\|\mathrm{~h}^{-\frac{1}{2}} \llbracket \boldsymbol{v}_{h} \rrbracket\right\|_{\mathcal{F}_{h}^{I \cup \Gamma}}^{2}-\delta C_{\mathrm{inv}} \mu_{-}^{-2}\left\|\nabla_{h} \times \boldsymbol{v}_{h}\right\|_{L^{2}(\Omega)^{3}}^{2} .
\end{aligned}
$$

Once again $\sqrt{x+y} \geq \frac{1}{\sqrt{2}}(\sqrt{x}+\sqrt{y})$ together with the boundedness $(2.2)$ of $\mu$ gives

$$
\begin{aligned}
\geq & \frac{1}{2}\left(\sqrt{1-a}\left[\mu_{+}^{-1}\left\|\nabla_{h} \times \boldsymbol{v}_{h}\right\|_{L^{2}(\Omega)^{3}}^{2}+\tau\left\|\mathrm{h}^{-\frac{1}{2}} \llbracket \boldsymbol{v}_{h} \rrbracket\right\|_{\mathcal{F}_{h}^{I \cup \Gamma}}^{2}\right]\right. \\
& \left.+\omega\left(\sigma_{-}^{2}-\left(a^{-1}-1\right) \omega^{2} \varepsilon_{+}^{2}\right)^{1 / 2}\left\|\boldsymbol{v}_{h}\right\|_{L^{2}(\Omega)^{3}}^{2}\right)+\frac{\omega \lambda \sqrt{\varepsilon_{0}}}{\sqrt{2 \mu_{0}}}\left\|\left(\boldsymbol{v}_{h}\right)_{T}\right\|_{L^{2}(\Sigma)^{3}}^{2}
\end{aligned}
$$




$$
\begin{aligned}
& -\delta^{-1}\left\|\mathrm{~h}^{-\frac{1}{2}} \llbracket \boldsymbol{v}_{h} \rrbracket\right\|_{\mathcal{F}_{h}^{I \cup I}}^{2}-\delta C_{\mathrm{inv}} \mu_{-}^{-2}\left\|\nabla_{h} \times \boldsymbol{v}_{h}\right\|_{L^{2}(\Omega)^{3}}^{2} \\
= & \left\|\nabla_{h} \times \boldsymbol{v}_{h}\right\|_{L^{2}(\Omega)^{3}}^{2}\left(\frac{\sqrt{1-a}}{2 \mu_{+}}-\delta C_{\mathrm{inv}} \mu_{-}^{-2}\right) \\
& +\left\|\boldsymbol{v}_{h}\right\|_{L^{2}(\Omega)^{3}}^{2} \frac{\omega\left(\sigma_{-}^{2}-\left(a^{-1}-1\right) \omega^{2} \varepsilon_{+}^{2}\right)^{1 / 2}}{2}+\left\|\left(\boldsymbol{v}_{h}\right)_{T}\right\|_{L^{2}(\Sigma)^{3}}^{2} \frac{\omega \lambda \sqrt{\varepsilon_{0}}}{\sqrt{2 \mu_{0}}} \\
& +\left\|\mathrm{h}^{-\frac{1}{2}} \llbracket \boldsymbol{v}_{h} \rrbracket\right\|_{\mathcal{F}_{h}^{I \cup I}}^{2}\left(\frac{\tau \sqrt{1-a}}{2}-\delta^{-1}\right) .
\end{aligned}
$$

We may choose $1>a>\frac{\omega^{2} \varepsilon_{+}^{2}}{\omega^{2} \varepsilon_{+}^{2}+\sigma_{-}^{2}}$, e.g., $a=\frac{\omega^{2} \varepsilon_{+}^{2}+\frac{\sigma_{-}^{2}}{2}}{\omega^{2} \varepsilon_{+}^{2}+\sigma_{-}^{2}}$ and obtain

$$
\begin{aligned}
= & \left\|\nabla_{h} \times \boldsymbol{v}_{h}\right\|_{L^{2}(\Omega)^{3}}^{2}\left(\frac{1}{2 \mu_{+}}\left(\frac{\sigma_{-}^{2}}{2\left(\omega^{2} \varepsilon_{+}^{2}+\sigma_{-}^{2}\right)}\right)^{\frac{1}{2}}-\delta C_{\mathrm{inv}} \mu_{-}^{-2}\right) \\
& +\left\|\boldsymbol{v}_{h}\right\|_{L^{2}(\Omega)^{3}}^{2} \frac{\omega}{2}\left(\sigma_{-}^{2}-\frac{\sigma_{-}^{2}}{2 \omega^{2} \varepsilon_{+}^{2}+\sigma_{-}^{2}} \omega^{2} \varepsilon_{+}^{2}\right)^{\frac{1}{2}}+\left\|\left(\boldsymbol{v}_{h}\right)_{T}\right\|_{L^{2}(\Sigma)^{3}}^{2} \frac{\omega \lambda \sqrt{\varepsilon_{0}}}{\sqrt{2 \mu_{0}}} \\
& +\left\|\mathrm{h}^{-1} \llbracket \boldsymbol{v}_{h} \rrbracket\right\|_{\mathcal{F}_{h}^{I \cup \Gamma}}^{2}\left(\frac{\tau}{2}\left(\frac{\sigma_{-}^{2}}{2\left(\omega^{2} \varepsilon_{+}^{2}+\sigma_{-}^{2}\right)}\right)^{\frac{1}{2}}-\delta^{-1}\right) \\
= & \left\|\nabla_{h} \times \boldsymbol{v}_{h}\right\|_{L^{2}(\Omega)^{3}}^{2}\left(\frac{\sigma_{-}}{2^{3 / 2} \mu_{+}\left(\omega^{2} \varepsilon_{+}^{2}+\sigma_{-}^{2}\right)^{1 / 2}}-\delta C_{\mathrm{inv}} \mu_{-}^{-2}\right) \\
& +\left\|\boldsymbol{v}_{h}\right\|_{L^{2}(\Omega)^{3}}^{2} \frac{\omega}{2}\left(\frac{\omega^{2} \varepsilon_{+}^{2} \sigma_{-}^{2}+\sigma_{-}^{4}}{2 \omega^{2} \varepsilon_{+}^{2}+\sigma_{-}^{2}}\right)^{\frac{1}{2}}+\left\|\left(\boldsymbol{v}_{h}\right)_{T}\right\|_{L^{2}(\Sigma)^{3}}^{2} \frac{\omega \lambda \sqrt{\varepsilon_{0}}}{\sqrt{2 \mu_{0}}} \\
& +\left\|\mathrm{h}^{-1} \llbracket \boldsymbol{v}_{h} \rrbracket\right\|_{\mathcal{F}_{h}^{I \cup \Gamma}}^{2}\left(\frac{\tau \sigma_{-}}{2^{3 / 2}\left(\omega^{2} \varepsilon_{+}^{2}+\sigma_{-}^{2}\right)^{1 / 2}}-\delta^{-1}\right) .
\end{aligned}
$$

Choose $0<\delta<\frac{\mu_{-}^{2} \sigma_{-}}{2^{3 / 2} C_{\text {inv }} \mu_{+}\left(\omega^{2} \varepsilon_{+}^{2}+\sigma_{-}^{2}\right)^{1 / 2}}$. e.g. $\delta=\frac{\mu_{-}^{2} \sigma_{-}}{2^{5 / 2} C_{\text {inv }} \mu_{+}\left(\omega^{2} \varepsilon_{+}^{2}+\sigma_{-}^{2}\right)^{1 / 2}}$ yields

$$
\begin{aligned}
= & \left\|\nabla_{h} \times \boldsymbol{v}_{h}\right\|_{L^{2}(\Omega)^{3}}^{2} \frac{\sigma_{-}}{2^{5 / 2} \mu_{+}\left(\omega^{2} \varepsilon_{+}^{2}+\sigma_{-}^{2}\right)^{1 / 2}} \\
& +\left\|\boldsymbol{v}_{h}\right\|_{L^{2}(\Omega)^{3}}^{2} \frac{\omega}{2}\left(\frac{\omega^{2} \varepsilon_{+}^{2} \sigma_{-}^{2}+\sigma_{-}^{4}}{2 \omega^{2} \varepsilon_{+}^{2}+\sigma_{-}^{2}}\right)^{\frac{1}{2}}+\left\|\left(\boldsymbol{v}_{h}\right)_{T}\right\|_{L^{2}(\Sigma)^{3}}^{2} \frac{\omega \lambda \sqrt{\varepsilon_{0}}}{\sqrt{2 \mu_{0}}} \\
& +\left\|\mathrm{h}^{-1} \llbracket \boldsymbol{v}_{h} \rrbracket\right\|_{\mathcal{F}_{h}^{I \cup \Gamma}}^{2}\left(\frac{\tau \sigma_{-}}{2^{3 / 2}\left(\omega^{2} \varepsilon_{+}^{2}+\sigma_{-}^{2}\right)^{1 / 2}}-\frac{2^{5 / 2} C_{\mathrm{inv}} \mu_{+}\left(\omega^{2} \varepsilon_{+}^{2}+\sigma_{-}^{2}\right)^{1 / 2}}{\mu_{-}^{2} \sigma_{-}}\right) .
\end{aligned}
$$

Hence, for all penalty parameters $\tau>\tau^{*}$ with $\tau^{*}$ defined by (3.17), e.g., choosing specifically $\tau=\frac{2^{3 / 2}\left(\omega^{2} \varepsilon_{+}^{2}+\sigma_{-}^{2}\right)^{1 / 2}}{\sigma_{-}}\left(\frac{2^{5 / 2} C_{\mathrm{inv}} \mu_{+}\left(\omega^{2} \varepsilon_{+}^{2}+\sigma_{-}^{2}\right)^{1 / 2}}{\mu_{-}^{2} \sigma_{-}}+\frac{\omega \lambda \sqrt{\varepsilon_{0}}}{\sqrt{2 \mu_{0}}}\right)$, we obtain

$$
\begin{aligned}
& \geq \min \left\{\frac{\sigma_{-}}{2^{5 / 2} \mu_{+}\left(\omega^{2} \varepsilon_{+}^{2}+\sigma_{-}^{2}\right)^{1 / 2}}, \frac{\omega}{2}\left(\frac{\omega^{2} \varepsilon_{+}^{2} \sigma_{-}^{2}+\sigma_{-}^{4}}{2 \omega^{2} \varepsilon_{+}^{2}+\sigma_{-}^{2}}\right)^{\frac{1}{2}}, \frac{\omega \lambda \sqrt{\varepsilon_{0}}}{\sqrt{2 \mu_{0}}}\right\}\left\|\boldsymbol{v}_{h}\right\|_{\mathrm{DG}}^{2} \\
& =\alpha_{\mathrm{DG}}(\omega)\left\|\boldsymbol{v}_{h}\right\|_{\mathrm{DG}}^{2},
\end{aligned}
$$

and everything is proven. 
TABLE B.1. Weak Form PDE and boundary conditions.

\begin{tabular}{|c|c|}
\hline \multicolumn{2}{|r|}{ Weak Form PDE, whole domain } \\
\hline \multirow[t]{3}{*}{ weak (1) } & 1/mu*rotE1*rotv1 - omega*omega*epsilon*E1*test(E1) \\
\hline & -i*omega*sigma*E1*test(E1) \\
\hline & -i*omega*sqrt(epsilon0)*Ja1*test(E1) \\
\hline weak $(2),(3)$ & accordingly \\
\hline \multicolumn{2}{|c|}{ Weak Contributions on Mesh Boundaries, whole domain } \\
\hline \multirow[t]{2}{*}{ Weak expression (1) } & -jumpE1 ${ }^{*} 1 / \mathrm{mu} *$ avrotv1 $-1 / \mathrm{mu} *$ avrotE1 ${ }^{*}$ jumpv1 \\
\hline & + tau/h*jumpE1*jumpv1 \\
\hline Weak expression $(2),(3)$ & accordingly \\
\hline \multicolumn{2}{|c|}{ Weak Contributions on Interior Block Boundaries, interior blocks } \\
\hline \multirow[t]{3}{*}{ Weak expression (1) } & $-($ ny*E3-nz*E2)*1/mu*rotv1 \\
\hline & $-1 / m u^{*} \operatorname{rotE1}($ ny*test(E3)-nz*test(E2)) \\
\hline & +tau/h*(ny*E3-nz*E2)*(ny*test(E3)-nz*test(E2)) \\
\hline Weak expression $(2),(3)$ & accordingly \\
\hline \multicolumn{2}{|r|}{ Exterior Boundary Conditions, outer boundary } \\
\hline Weak expression (4) & - $\left(\mathrm{g} 1 * \mathrm{vT} 1+\mathrm{g} 22^{*} \mathrm{vT} 2+\mathrm{g} 3 * \mathrm{vT} 3\right) / \mathrm{mu} 0$ \\
\hline \multirow[t]{2}{*}{ Weak expression (5) } & -i*omega*lambda ${ }^{*}$ sqrt(epsilon0/mu0) \\
\hline & $*\left(\mathrm{ET} 1{ }^{*} \mathrm{vT} 1+\mathrm{ET} 2{ }^{*} \mathrm{vT} 2+\mathrm{ET} 3{ }^{*} \mathrm{vT} 3\right)$ \\
\hline
\end{tabular}

TABle B.2. Parameters.

\begin{tabular}{lcc}
\hline Global Parameters & Expression & Description \\
\hline omega & $1 \times 1 \mathrm{e} 9$ & frequency, $\omega \in[1 \cdot 1 \mathrm{e} 9,50 \cdot 1 \mathrm{e} 9]$ \\
epsilon0 & $8.854 \times 1 \mathrm{e}-12$ & electric permittivity in vacuum \\
$\mathrm{Ja} 1$ & $1,00 \mathrm{E}+004$ & electric current density, first entry \\
$\mathrm{Ja} 2$ & $1,00 \mathrm{E}+004$ & electric current density, second entry \\
$\mathrm{Ja} 3$ & $1,00 \mathrm{E}+004$ & electric current density, third entry \\
mu0 & $4 \times \mathrm{pi} \times 1 \mathrm{e}-7$ & magnetic permeability in vacuum \\
tau & $1000 / \mathrm{mu} 0$ & penalty parameter for dG formulation \\
lambda & 1 & $\lambda>0$, intensity of impedance \\
\hline
\end{tabular}

\section{Appendix B. Description of the COMSOL MOdel}

In order to enable reproducibility, we provide details about the COMSOL model which we used for our numerical experiments. We use the Weak Form PDE from Mathematics $\rightarrow$ PDE interfaces and define three Dependent variables E1, E2, and E3. For reasons of compactness we only show the first entry here when dealing with vectors or multiple similar expressions.

Table B.1 shows the weak expressions (1), (2) and (3) defined by the weak form PDE node on the whole domain as well as jump and average terms on the mesh boundaries and the contributions on the interior block boundaries to the weak formulation. Parameters and Variables are indicated in Tables B.2 and B.3, respectively. In particular, the used source functions $\boldsymbol{J}_{\boldsymbol{a}}$ and $\boldsymbol{g}$ can be found there. A physics-controlled mesh was used, the Element size varied from coarser to finer. In all cases we used Discontiuous Lagrange as shape function type. 
TABLE B.3. Variables.

\begin{tabular}{|c|c|c|}
\hline Variables & Expression & Description \\
\hline rotE1 & E3y-E2z & $\nabla_{h} \times E$, first entry \\
\hline rotv1 & test(E3y)-test(E2z) & $\nabla_{h} \times v$, first entry \\
\hline jumpE1 & dny*down(E3)-dnz*down(E2) +uny*up(E3)-unz*up(E2) & $\llbracket E \rrbracket$ on $\mathcal{F}_{h}^{I}$, first entry \\
\hline jumpv1 & dny*test(down(E3))-dnz*test(down(E2)) + uny*test(up(E3))-unz*test(up(E2) & )) $v v \rrbracket$ on $\mathcal{F}_{h}^{I}$, first entry \\
\hline avrotE1 & $0.5^{*}(\mathrm{up}(\mathrm{E} 3 \mathrm{y})-\mathrm{up}(\mathrm{E} 2 \mathrm{z})+$ down$(\mathrm{E} 3 \mathrm{y})-\mathrm{down}(\mathrm{E} 2 \mathrm{z}))$ & $\left\{\left\{\nabla_{h} \times E\right\}\right.$, firs \\
\hline avrotv1 & $0.5^{*}(\operatorname{test}(\mathrm{up}(\mathrm{E} 3 \mathrm{y}))-\operatorname{test}(\mathrm{up}(\mathrm{E} 2 \mathrm{z}))+\operatorname{test}(\operatorname{down}(\mathrm{E} 3 \mathrm{y}))-\operatorname{test}(\operatorname{down}(\mathrm{E} 2 \mathrm{z})))$ & $\left\{\left\{\nabla_{h} \times v\right\}\right.$, first entry \\
\hline ET1 & $\mathrm{nz}{ }^{*} \mathrm{E} 1 * \mathrm{nz}-\mathrm{nx} * \mathrm{E} 3 * \mathrm{nz}-\mathrm{nx} * \mathrm{E} 2 * \mathrm{ny}+\mathrm{ny} * \mathrm{E} 1 * \mathrm{ny}$ & $E_{T}$, first entry \\
\hline $\mathrm{vT} 1$ & nz*test $(\mathrm{E} 1) * \mathrm{nz}-\mathrm{nx} * \operatorname{test}(\mathrm{E} 3) * \mathrm{nz}-\mathrm{nx} * \operatorname{test}(\mathrm{E} 2) * \mathrm{ny}+\mathrm{ny}{ }^{*} \operatorname{test}(\mathrm{E} 1) * \mathrm{ny}$ & $v_{T}$, first entry \\
\hline $\mathrm{mu}$ & mu0*(1+distance_midpo & magnetic \\
\hline sigma & $0.01 *\left(1+0.5 * x^{\wedge} 2\right)$ & electric cond \\
\hline epsilon & epsilon $0 *(1+$ distance_000) & electric per \\
\hline oint & $\operatorname{sqrt}\left((0.5-x)^{\wedge} 2+(0.5-y)^{\wedge} 2+(0.5-z)^{\wedge} 2\right)$ & $\left\|\boldsymbol{x}-\left(\frac{1}{2}, \frac{1}{2}, \frac{1}{2}\right)^{T}\right\|$ \\
\hline ance_000 & $\operatorname{sqrt}\left(x^{\wedge} 2+y^{\wedge} 2+z\right.$ & \\
\hline $\mathrm{g} 1, \mathrm{~g} 2, \mathrm{~g} 3$ & $\mathrm{t} 1 \mathrm{x}, \mathrm{t} 1 \mathrm{y}, \mathrm{t} 1 \mathrm{z}$ & $g \in L_{t}^{2}(\Sigma)^{3}$ \\
\hline
\end{tabular}

\section{REFERENCES}

[1] R.A. Adams and J.J.F. Fournier, Sobolev Spaces. Academic Press, New York (2003).

[2] A. Alonso and A. Valli, An optimal domain decomposition preconditioner for low-frequency time-harmonic Maxwell equations. Math. Comput. 68 (1999) 607-631.

[3] P.R. Amestoy, I.S. Duff, J. Koster and J.-Y. L'Excellent, A fully asynchronous multifrontal solver using distributed dynamic scheduling. SIAM J. Matrix Anal. Appl. 23 (2001) 15-41.

[4] P.R. Amestoy, A. Guermouche, J.-Y. L'Excellent and S. Pralet, Hybrid scheduling for the parallel solution of linear systems. Parallel Computing 32 (2006) 136-156.

[5] D.N. Arnold, F. Brezzi, B. Cockburn and L.D. Marini, Unified analysis of discontinuous Galerkin methods for elliptic problems. SIAM J. Numer. Anal. 39 (2001) 1749-1779.

[6] C. Bernardi and V. Girault, A local regularization operator for triangular and quadrilateral finite elements. SIAM J. Numer. Anal. 35 (1998) 1893-1916.

[7] P. Binev, A. Cohen, W. Dahmen, R. DeVore, G. Petrova and P. Wojtaszczyk, Convergence Rates for Greedy Algorithms in Reduced Basis Methods. SIAM J. Math. Anal. 43 (2011) 1457-1472.

[8] A. Buffa, M. Costabel and D. Sheen, On traces for $H(\operatorname{curl}, \Omega)$ in Lipschitz domains. J. Math. Anal. Appl. 276 (2002) 845-867.

[9] A. Buffa and R. Hiptmair, Galerkin boundary element methods for electromagnetic scattering. In Topics in Computational Wave Propagation. Vol. 31 of Lect. Notes Comput. Sci. Eng. Springer Berlin Heidelberg (2003) 83-124.

[10] Y. Chen, J.S. Hesthaven, Y. Maday and J. Rodríguez, Improved successive constraint method based a posteriori error estimate for reduced basis approximation of 2D Maxwell's problem. ESAIM: M2AN 43 (2009) 1099-1116.

[11] Y. Chen, J.S. Hesthaven, Y. Maday and J. Rodríguez, Certified reduced basis methods and output bounds for the harmonic Maxwell's equations. SIAM J. Sci. Comput. 32 (2010) 970-996.

[12] P. Clément, Approximation by finite element functions using local regularization. RAIRO Anal. Numer. 9 (1975) $77-84$.

[13] J.L. Eftang, A.T. Patera and E.M. Rønquist, An " $h p$ " certified reduced basis method for parametrized elliptic partial differential equations. SIAM J. Sci. Comput. 32 (2010) 3170-3200.

[14] J.L. Eftang, D.J. Knezevic and A.T. Patera, An " $h p$ " certified reduced basis method for parametrized parabolic partial differential equations. Math. Comput. Model. Dyn. Syst. 17 (2011) 395-422.

[15] M. Fares, J.S. Hesthaven, Y. Maday and B. Stamm, The reduced basis method for the electric field integral equation. J. Comp. Phys. 230 (2011) 5532-5555.

[16] M. Ganesh, J.S. Hesthaven and B. Stamm, A reduced basis method for electromagnetic scattering by multiple particles in three dimensions. J. Comp. Phys. 231 (2012) 7756-7779.

[17] G.N. Gatica and S. Meddahi, Finite element analysis of a time harmonic Maxwell problem with an impedance boundary condition. IMA J. Numer. Anal. 32 (2012) 534-552.

[18] V. Girault and P.-A. Raviart, Finite Element Methods for Navier-Stokes Equations: Theory and Algorithms. Springer (1986).

[19] M.W. Hess and P. Benner, Fast Evaluation of Time-Harmonic Maxwell's Equations Using the Reduced Basis Method. IEEE Trans. Microw. Theory Tech. 61 (2013) 2265-2274.

[20] J.S. Hesthaven, B. Stamm and S. Zhang, Certified reduced basis method for the electric field integral equation. SIAM J. Sci. Comput. 34 (2012) A1777-A1799. 
[21] R. Hiptmair, A. Moiola and I. Perugia, Error analysis of Trefftz-discontinuous Galerkin methods for the time-harmonic Maxwell equations. Math. Comput. 82 (2013) 247-268.

[22] P. Houston, I. Perugia, A. Schneebeli and D. Schötzau, Interior penalty method for the indefinite time-harmonic Maxwell equations. Numer. Math. 100 (2005) 485-518.

[23] D.B.P. Huynh, G. Rozza, S. Sen and A.T. Patera, A successive constraint linear optimization method for lower bounds of parametric coercivity and inf-sup stability constants. C. R. Math. Acad. Sci. Paris Series I 345 (2007) 473-478.

[24] S. Kaulmann, M. Ohlberger and B. Haasdonk, A new local reduced basis discontinuous Galerkin approach for heterogeneous multiscale problems. C. R. Math. Acad. Sci. Paris 349 (2011) 1233-1238.

[25] W. McLean, Strongly Elliptic Systems and Boundary Integral Equations. Cambridge University Press, Cambridge (2000).

[26] P. Monk, Finite Element Methods for Maxwell's Equations. Numerical Mathematics and Scientific Computation. Oxford University Press, Oxford (2003).

[27] F. Negri, G. Rozza, A. Manzoni and A. Quarteroni, Reduced basis method for parametrized elliptic optimal control problems. SIAM J. Sci. Comput. 35 (2013) A2316 - A2340.

[28] I. Perugia and D. Schötzau, The $h p$-local discontinuous Galerkin method for low-frequency time-harmonic Maxwell equations. Math. Comput. 72 (2003) 1179-1214.

[29] J. Pomplun and F. Schmidt, Accelerated a posteriori error estimation for the reduced basis method with application to 3D electromagnetic scattering problems. SIAM J. Sci. Comput. 32 (2010) 498-520.

[30] G. Rozza, D.B. Phuong Huynh and A.T. Patera, Reduced basis approximation and a posteriori error estimation for affinely parametrized elliptic coercive partial differential equations: Application to transport and continuum mechanics. Arch. Comput. Methods. Eng. 15 (2008) 229-275.

[31] D. Sármány, F. Izsák and J.J.W. van der Vegt, Optimal penalty parameters for symmetric discontinuous Galerkin discretisations of the time-harmonic Maxwell equations. J. Sci. Comput. 44 (2010) 219-254.

[32] T. Warburton and J.S. Hesthaven, On the constants in $h p$-finite element trace inverse inequalities. Comput. Methods Appl. Mech. Engrg. 192 (2003) 2765-2773. 